ACCEPTED MANUSCRIPT

\title{
Lightweight mechanical metamaterials designed using hierarchical truss elements
}

To cite this article before publication: Luke Mizzi et al 2020 Smart Mater. Struct. in press https://doi.org/10.1088/1361-665X/aba53c

\section{Manuscript version: Accepted Manuscript}

Accepted Manuscript is "the version of the article accepted for publication including all changes made as a result of the peer review process, and which may also include the addition to the article by IOP Publishing of a header, an article ID, a cover sheet and/or an 'Accepted

Manuscript' watermark, but excluding any other editing, typesetting or other changes made by IOP Publishing and/or its licensors"

This Accepted Manuscript is @ 2020 IOP Publishing Ltd.

During the embargo period (the 12 month period from the publication of the Version of Record of this article), the Accepted Manuscript is fully protected by copyright and cannot be reused or reposted elsewhere.

As the Version of Record of this article is going to be / has been published on a subscription basis, this Accepted Manuscript is available for reuse under a CC BY-NC-ND 3.0 licence after the 12 month embargo period.

After the embargo period, everyone is permitted to use copy and redistribute this article for non-commercial purposes only, provided that they adhere to all the terms of the licence https://creativecommons.org/licences/by-nc-nd/3.0

Although reasonable endeavours have been taken to obtain all necessary permissions from third parties to include their copyrighted content within this article, their full citation and copyright line may not be present in this Accepted Manuscript version. Before using any content from this article, please refer to the Version of Record on IOPscience once published for full citation and copyright details, as permissions will likely be required. All third party content is fully copyright protected, unless specifically stated otherwise in the figure caption in the Version of Record.

View the article online for updates and enhancements. 


\title{
Lightweight Mechanical Metamaterials designed using Hierarchical Truss Elements
}

\author{
Luke Mizzi $^{1 *}$, Andrea Spaggiari ${ }^{1}$ \\ ${ }^{1}$ Department of Engineering Sciences and Methods, University of Modena and Reggio Emilia, Reggio Emilia, \\ Italy
}

*corresponding author email: luke.mizzi@unimore.it

\begin{abstract}
Rotating unit systems constitute one of the main classes of auxetic metamaterials. In this work, a new design procedure for lightweight auxetic systems based on this deformation mechanism is proposed through the implementation of a hierarchical triangular truss network in place of a full block of material for the rotating component of the system. Using numerical simulations in conjunction with experimental tests on $3 \mathrm{D}$ printed prototypes, the mechanical properties of six types of auxetic structures, which include a range of rotating polygons and chiral honeycombs, were analysed under the application of small tensile loads. The results obtained show that there is almost no difference in the Poisson's ratios obtained from the regular, full structures and the ones made from triangular truss systems despite the latter, in some cases, being $80 \%$ lighter than the former. This indicates that these systems could be ideal candidates for implementation in applications requiring lightweight auxetic metamaterial systems such as in the aerospace industry.
\end{abstract}

Keywords: Auxetics, Mechanical Metamaterials, Truss Systems, Mechanical Properties, 3D Printing, Lightweight Systems

\section{Introduction}

Mechanical metamaterials are systems whose mechanical properties are derived primarily from their structural framework and geometric design. These systems have attracted a great deal of interest in the fields of material science and engineering due to their capability to exhibit a wide range of mechanical properties including unusual properties such as a negative Poisson's ratio (auxeticity) [1-4]. These 'negative' mechanical properties are typically accompanied by other derived unusual characteristics such as synclastic curvature [5], increased indentation 
resistance [6] and enhanced acoustic performance [7] which makes them particularly suitable for a variety of applications.

One of the main classes of auxetic mechanical metamaterials is that of rotating unit systems. These metamaterials are typically made up of rigid or semi-rigid blocks of material which rotate relative to each other when the system is loaded. The earliest and most commonly known example of these systems is the rotating square system [8], which as its name implies, is made up of square building blocks. In its ideal form, the rotating square system has an isotropic Poisson's ratio of -1 and deforms solely via rotation of the rigid square units. This 'rotating mechanism' is not limited to square systems only and can also be observed in a number of 2D and 3D systems including rotating triangles $[9,10]$, rectangles [11], parallelograms $[12,13]$ and cubes [14] amongst others. Chiral metamaterial structures [15-18] also possess this 'rotating' characteristic. However, unlike the former class of systems, the rotating units in chiral honeycombs are connected to each other through ligaments rather than joints, with the ligaments undergoing deformation while the 'rigid' chiral unit rotates. Similarly to rotating rigid unit structures, there is a wide range of possible geometries which may be obtained for these systems depending on the shape of the chiral unit and their mode of connection and thus these systems also have the potential to exhibit an extremely large spectrum of mechanical properties.

In both of these cases, the efficacy of the 'rotating mechanism' depends primarily on the rigidity/shape retention of the rotating unit. The most common method employed to enforce this in chiral and rotating rigid unit structures is by typically designing the rotating units as a solid block of material. This makes them extremely stiff and thus the bulk of deformation of the structure occurs at the joint and/or ligament regions of the unit upon loading, leading to rotation of the unit. However, this is not the only method through which rigidity of the rotating unit may be achieved. Indeed, one may argue that such a method is extremely inefficient since the bulk of material and hence, mass, within the system remains undeformed during loading and is being 'wasted' solely to maintain the rigidity of the rotating unit. This could be a significant disadvantage in some cases which could discourage the use of these metamaterial geometries for applications where the material composition of the metamaterial is extremely costly or lightweight structures are required. In some cases, such as in chiral honeycombs made from circular nodes, the problem has been tackled by employing hollow chiral nodes [19-22]. However, this approach has its own limitations since in order to ensure that a high negative Poisson's ratio is retained, chiral nodes must have a suitably small radius and appropriate 


\section{Methodology} as to remain rigid.

In view of this, in this work, a study aimed at designing lightweight metamaterial systems based on rotating unit structures whilst maintaining the auxetic potential of these systems, was conducted. The investigation was primarily focused on exploring the merits of introducing hierarchical triangular truss-like components within the rotating unit and analysing the effects of these structural frameworks on the mechanical properties of the studied systems. Previous studies on other hierarchical auxetic systems have shown that the introduction of hierarchy in such structures may be used to obtain enhanced mechanical properties and/or provide structural stability through smart design [23-30]. Hierarchical structures, which are defined as structures which are themselves made from structures [31], are not limited solely to fractal or fractal-like systems $[25,32,33]$, but may also include the use of different geometries and mechanisms at separate hierarchical levels aimed at conferring additional enhanced properties to the system. In order to obtain a complete picture of this effect, a number of $2 \mathrm{D}$ metamaterial geometries with varying degrees of symmetry were investigated using Finite Element (FE) Analysis and experimental tests on $3 \mathrm{D}$ printed models.

A pictorial representation of the $2 \mathrm{D}$ metamaterial geometries which were considered in this study is shown in Figure 1. The systems selected for this study were the rotating triangles [9], rotating squares [8], Type I rotating rectangles [11], Type I $\beta$ rotating parallelograms [12], antitetrachiral [17] and hexachiral [15] honeycombs. These metamaterial geometries were chosen since they encompass the main systems in the classes of chiral and rotating rigid unit structures, i.e. three polygonal rotating unit shapes; triangles, quadrilaterals and hexagons, as well as varying degrees of symmetry in the case of quadrilaterals in the form of squares, rectangles and parallelograms. In addition, the two forms of chiral honeycombs chosen for investigation are both the most well-known examples of anti-chiral and chiral honeycombs respectively. 


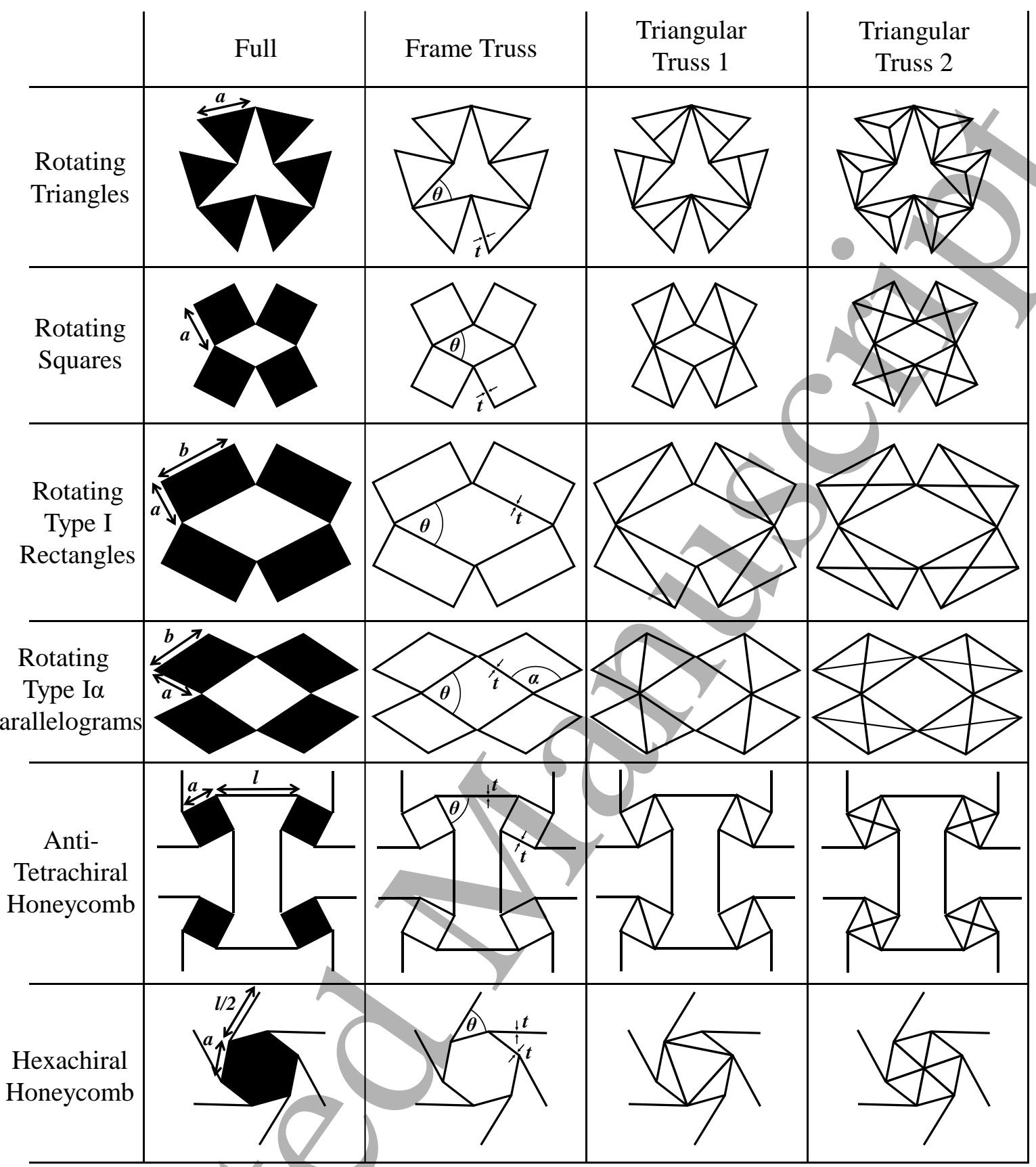

Figure 1: Pictorial representation of the four forms (Full, Frame Truss, Triangular Truss 1 and Triangular Truss 2) of the six auxetic metamaterial geometries investigated here including the geometric parameters used to define them.

Four cases were considered for each of these metamaterial geometries (see Figure 1). The first case was the 'full' structure where the rotating unit is completely filled with material. This is the case most commonly studied in literature and is considered to be the standard method for designing these metamaterials. The second case is the 'frame truss' geometry where the rotating unit is designed only as a frame made up of beam-like ligaments. In the third and fourth cases, the rotating unit is reinforced by the introduction of additional ligaments specifically designed to form triangular components. As shown in Figure 1, the third case, labelled as 
'Triangular Truss 1', is the geometrical configuration which permits the introduction of the minimum amount of additional triangular components to the rotating unit polygon, i.e. two in the case of the triangle and the quadrilaterals and four in the case of the hexagon. On the other hand, the fourth case, hereby designated as 'Triangular Truss 2', denotes the configuration which introduces the same number of triangular components as the number of sides of the rotating polygon, i.e. three, four and six in the cases of the triangles, quadrilaterals and hexagons respectively. Through this design, the rotating unit retains its original level of symmetry.

The simulations were conducted on the ANSYS16 Finite Element software using quadratic PLANE183 elements at linear plane-stress conditions. For each of the four cases mentioned previously, five different ligament thicknesses, $t$, were used, with the ligament thickness of the rotating unit frames being equal to that of the ligaments connecting the rotating units in the case of the chiral honeycombs. The range of thicknesses chosen for each structure were specifically chosen to cover thin, long ligaments, which behave predominantly as EulerBernoulli beams and thick, stubby ones which whose behaviour may be more accurately described by Timoshenko's beam theory. In addition, a number of geometric configurations were considered for each of these cases, with at least one where the mechanism is close to its fully-closed form, i.e. $\theta \rightarrow 0^{\circ}$, and another one where the mechanism in question is near its theoretical fully opened state, $\theta_{\max }$. Obviously, the actual values of $\theta$ depend entirely on the geometry in question, with each geometry having a different possible $\theta_{\max }$. All other parameters (see Figure 1) were kept constant for each geometry and were set as follows:

Table 1: Parameters of the systems simulated in this study. Note that the parameter $\alpha$ is only a variable in the case of the rotating parallelogram system; in the other geometries it is constrained to a single value by the symmetry of the rotating unit, i.e. it is $60^{\circ}$ for the rotating triangle system, $90^{\circ}$ for the other rotating quadrilateral systems and $120^{\circ}$ for the hexachiral system. In addition to these parameters, each of these systems were constructed according to the four forms shown in Figure 1.

\begin{tabular}{|c|c|c|c|c|c|c|c|c|}
\hline Geometry & $\boldsymbol{a}$ & $\boldsymbol{b}$ & $\boldsymbol{l}$ & $\boldsymbol{\alpha}$ & $\boldsymbol{r}$ & $\boldsymbol{o}$ & $\boldsymbol{\theta}$ & $\boldsymbol{t}$ \\
\hline $\begin{array}{c}\text { Rotating } \\
\text { Triangle }\end{array}$ & 30 & N/A & N/A & N/A & 0.2 & $\frac{a}{20}$ & $30^{\circ} \ldots 90^{\circ}$ & $\frac{a}{48} \ldots \frac{a}{8}$ \\
\hline $\begin{array}{c}\text { Rotating } \\
\text { Square }\end{array}$ & 30 & N/A & N/A & N/A & 0.2 & $\frac{a}{20}$ & $20^{\circ} \ldots 80^{\circ}$ & $\frac{a}{48} \ldots \frac{a}{8}$ \\
\hline $\begin{array}{l}\text { Rotating } \\
\text { Rectangle }\end{array}$ & 30 & 45 & N/A & N/A & 0.2 & $\frac{a}{20}$ & $28^{\circ} \ldots 80^{\circ}$ & $\frac{a}{48} \ldots \frac{a}{8}$ \\
\hline
\end{tabular}




\begin{tabular}{|c|c|c|c|c|c|c|c|c|}
\hline $\begin{array}{c}\text { Rotating } \\
\text { Parallelogram }\end{array}$ & 30 & 45 & N/A & $120^{\circ}$ & 0.2 & $\frac{a}{20}$ & $20^{\circ} \ldots 106^{\circ}$ & $\frac{a}{48} \ldots \frac{a}{8}$ \\
\hline $\begin{array}{c}\text { Anti- } \\
\text { Tetrachiral }\end{array}$ & 30 & N/A & 60 & N/A & 0.2 & $\frac{a}{20}$ & $20^{\circ} \ldots 90^{\circ}$ & $\frac{a}{48} \ldots \frac{a}{8}$ \\
\hline Hexachiral & 30 & N/A & 36 & N/A & 0.2 & $\frac{a}{20}$ & $20^{\circ} \ldots 60^{\circ}$ & $\frac{a}{48} \ldots \frac{a}{8}$ \\
\hline
\end{tabular}

While the parameters $a, b, l, \alpha, \theta$ and $t$, shown in Figure 1, define the main overall geometric parameters of metamaterial systems, $o$ and $r$ define the geometry of the joint regions. As shown in Figure 2, two types of joint are present in these systems and in each case, the parameter $r$ defines the radius of the fillet geometry which is used to eliminate the formation of sharp corners within the system. For the rotating polygon systems (Figure 2a), the joint is made up of two rotating blocks which 'overlap' each other. The degree of overlap, $o$, is defined as the thickness of the joint in the direction normal to that of the overlapping corners of the rotating unit. The example shown in Figure 2a represents a rotating square or rectangle system, where the internal angle $\alpha$ of the rotating unit is $90^{\circ}$. On the other hand, in the case of the chiral and anti-chiral systems (see Figure 2b), the amount of additional material at the joint is defined only by $r$. Here it is important to point out that the introduction of a fillet at the joint does not necessarily mean that additional material is added - this is only the case if the angle in question is less than $180^{\circ}$. Otherwise it results in a loss of /material as shown in Figure $\mathbf{2 b}$.

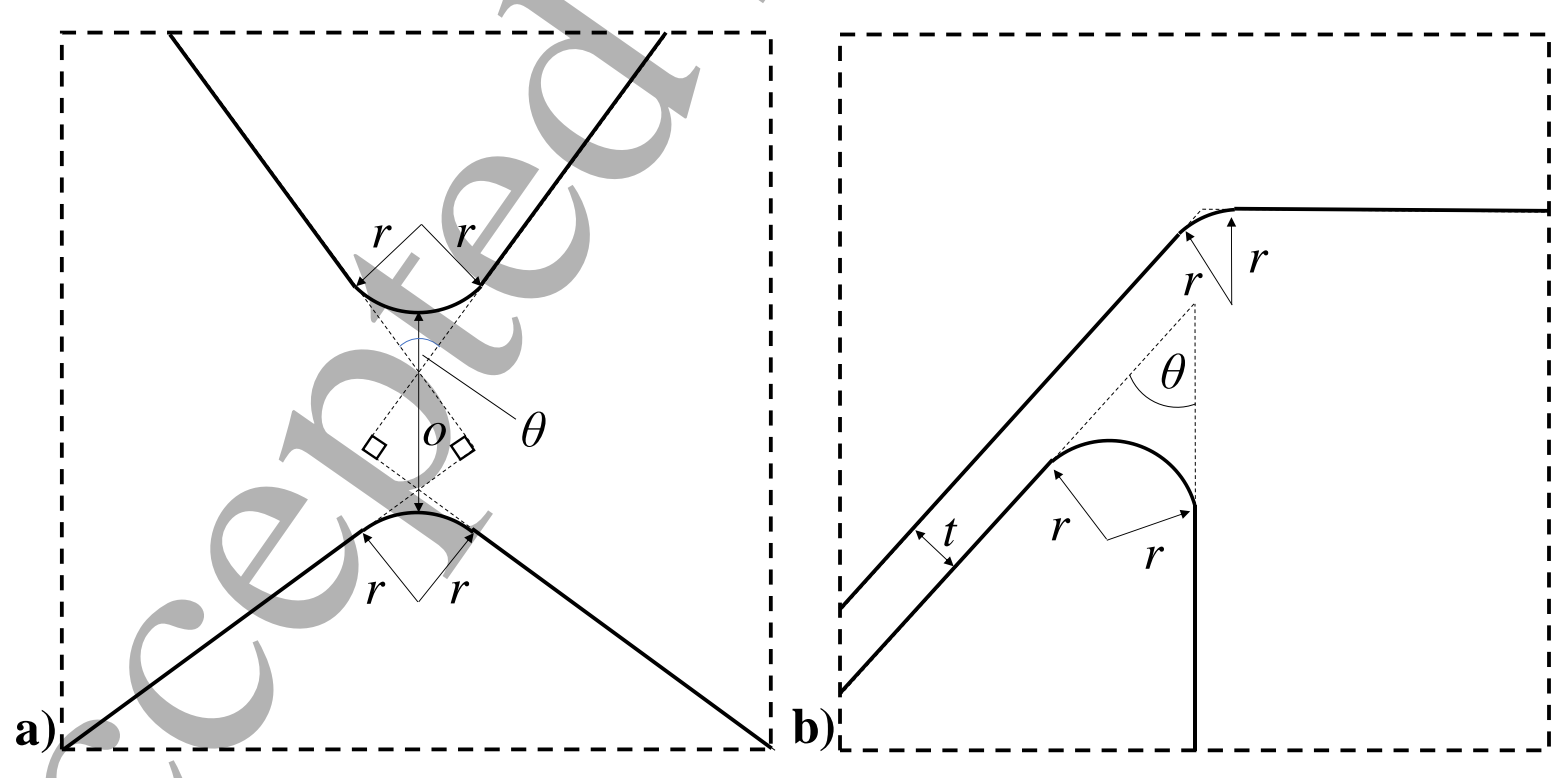

Figure 2: Diagram showing how the joints of (a) the rotating rigid unit systems and (b) the chiral and antichiral systems were constructed. Note that while in (a) material is added at the joints, in (b) a significant 
amount of material is added from one side of the joint while a small amount is subtracted from the opposite side as a result of the implementation of the fillet geometry.

Each system was simulated as a single unit cell using periodic boundary conditions for uniaxial tensile loading in the $x$ - and $y$-directions respectively. The periodic boundary conditions were implemented through the use of constraint equations and fixes as in the method detailed in [34] and deformation was induced through the application of forces on the edge nodes of the unit cell. Since we are primarily concerned with the small strain behaviour of these systems the simulations were computed using a linear solver. A Young's Modulus of $1.68 \mathrm{GPa}$ and a Poisson's ratio of 0.3 were used to describe the material properties of the system.

In addition to the linear simulations, one set of rotating square geometries were manufactured using a Formlabs2 3D printer using the Formlabs Tough Resin and loaded in the $y$-direction with a small tensile strain using a tensile loading device. These systems were designed according to the following specifications: $a=17 \mathrm{~mm}, t=0.77 \mathrm{~mm}, o=0.85 \mathrm{~mm}, r=0.15 \mathrm{~mm}$ and $\theta=48^{\circ}$, and consisted of $3 \times 3$ repeating unit cells as shown in Figure 3. The deformation of the central representative unit cell was evaluated using Digital Image Correlation (DIC) using the Istra3 $\mathrm{D}^{\circledR}$ software where two points on each of the four external boundaries of representative unit cell were tracked in order to determine the engineering strain in the $x$ - and $y$-directions. The material used has an intrinsic linear Young's modulus of $1.68 \mathrm{GPa}$ [35] and a full stress-strain plot can be found in [36,37]. Nonlinear FE simulations on systems corresponding to the $3 \mathrm{D}$ printed prototypes were also conducted, using boundary conditions equivalent to these of the experimental tensile loading machine.
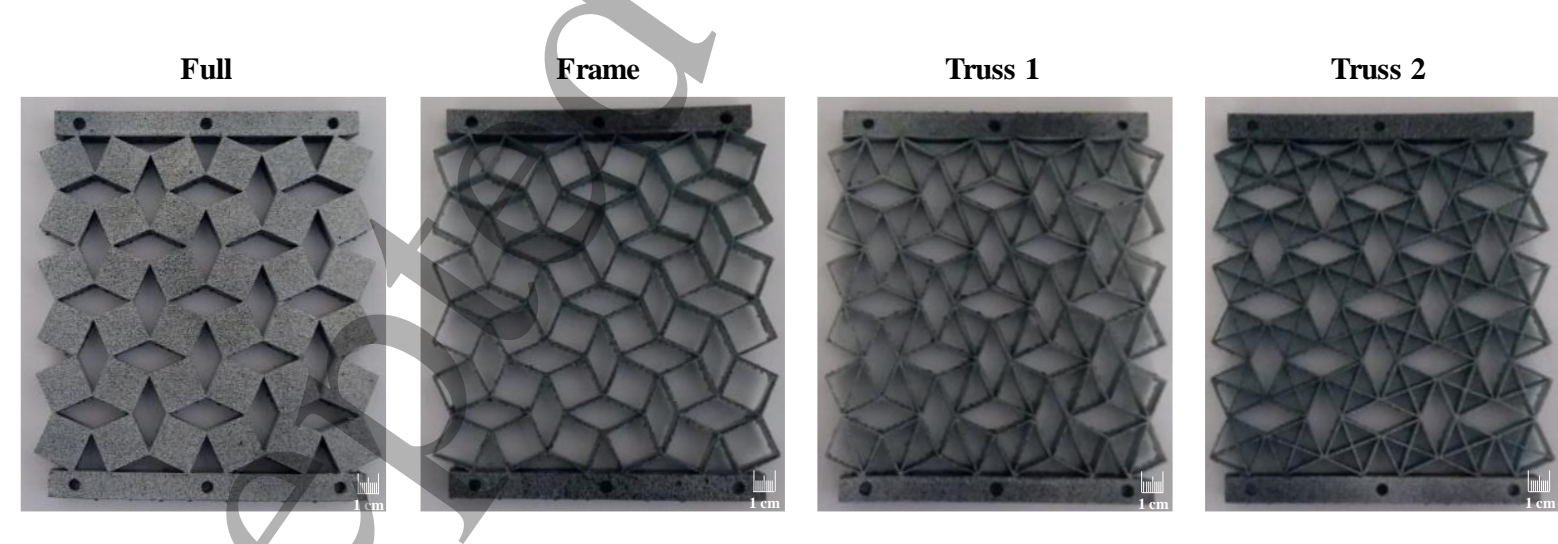

Figure 3: Images showing the four 3D printed rotating square systems. The systems were spray-painted after printing in order to generate the speckle pattern required for the DIC analysis. 


\section{Results and Discussion}

The results obtained for the linear Finite Element simulations of each geometry as constructed with the four types of internal geometry shown in Figure $\mathbf{1}$ are presented in this section followed by the experimental results.

\section{$\underline{\text { 3.1 Linear Finite Element Simulations }}$}

\section{Rotating Triangles}

Plots showing the Poisson's ratios and effective Young's moduli obtained for the rotating triangle systems with a ligament thickness of $a / 48$ (the smallest thickness) for loading in the $x$ direction are shown in Figure 4. The effective Young's modulus, $E_{\text {eff, }}$ is defined as the ratio of the measured Young's modulus of the geometry, $E^{*}$, in comparison to the material Young's modulus, $E_{m a t}$, i.e. $E_{\text {eff }}=\frac{E^{*}}{E_{\text {mat }}}$.

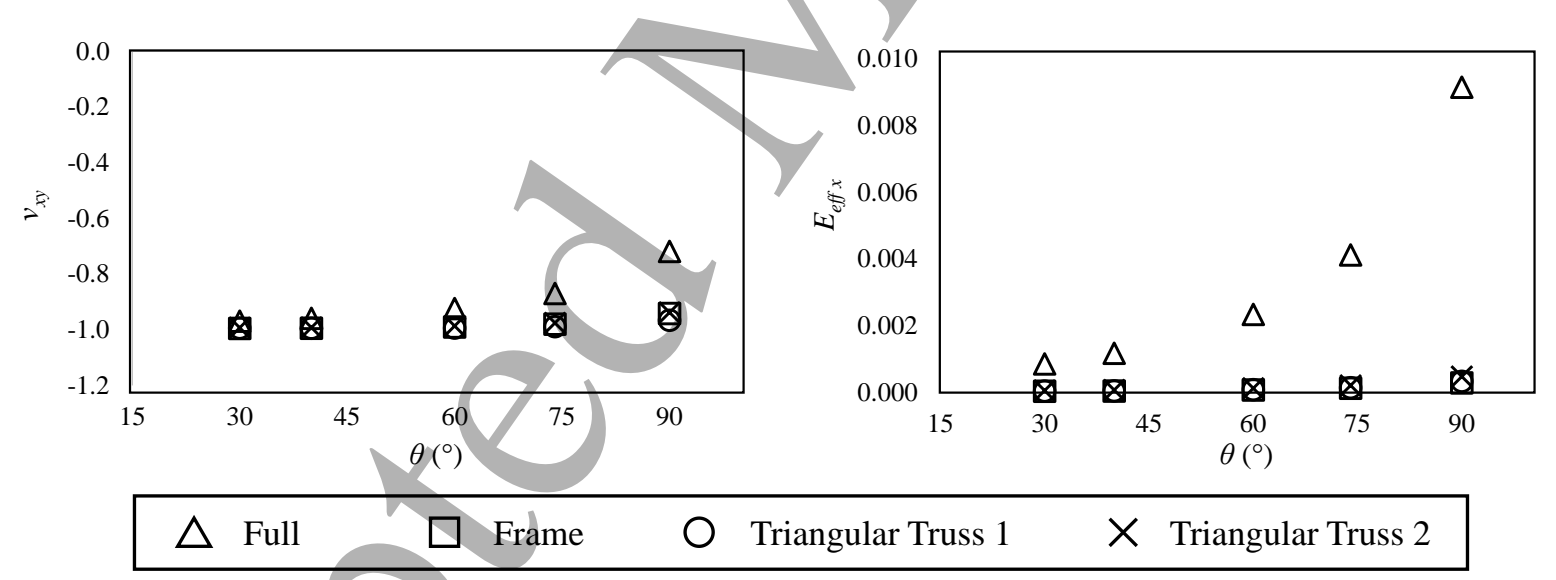

Figure 4: Plots showing the Poisson's ratio and effective Young's moduli for rotating triangle systems with $t=a / 48$ loaded in the $x$-direction.

It is evident from Figure 4 that the internal structure of the rotating triangle system has a significant effect on the mechanical properties of the overall system, particularly in the case of systems with large $\theta$ values. While it is observed that the Poisson's ratio becomes less negative as $\theta$ increases for all systems, this effect is especially pronounced in the case of the full systems where the Poisson's ratio goes from $c a$. -1 for the system where $\theta=30^{\circ}$ to $c a$. -0.7 for $\theta=90^{\circ}$. On the other hand, for the frame and triangular truss systems, this change is much less drastic. 
This decrease in auxeticity is typically observed for rotating rigid unit systems as the system approaches its fully open conformation (in this case it is achieved when $\theta$ becomes equal $120^{\circ}$ ), however it appears that the frame and triangular truss systems are not as adversely affected by this factor as their full counterparts. In full systems, this usually occurs due to the fact that the effective Young's modulus of the rotating rigid unit mechanism increases as $\theta$ increases [9] and thus other mechanisms which are not conducive to enhancing the auxetic behaviour of the system such as localized stretching of the joint regions come into play. This is evident from Figure 5, where it is shown that for the full systems the stress is concentrated solely at the joint regions. However, in the truss systems the stress is distributed throughout the ligaments and the joints, with the former undergoing flexure. This results in the 'rotating unit' component of the system losing its rigidity, however unlike localized 'stretching' of the joints, this deformation mechanism does not result in a reduction of the auxeticity of the system due to the triangulation of the truss components. This effect, where the ligaments bend concurrently, has been observed previously in a number of auxetic systems made from ligaments arranged in a triangular configuration and can be considered an auxetic mechanism in its own right $[38,39]$. This deformation mechanism also accounts for the large difference in the effective Young's modulus observed between the full and truss systems (see Table 2).
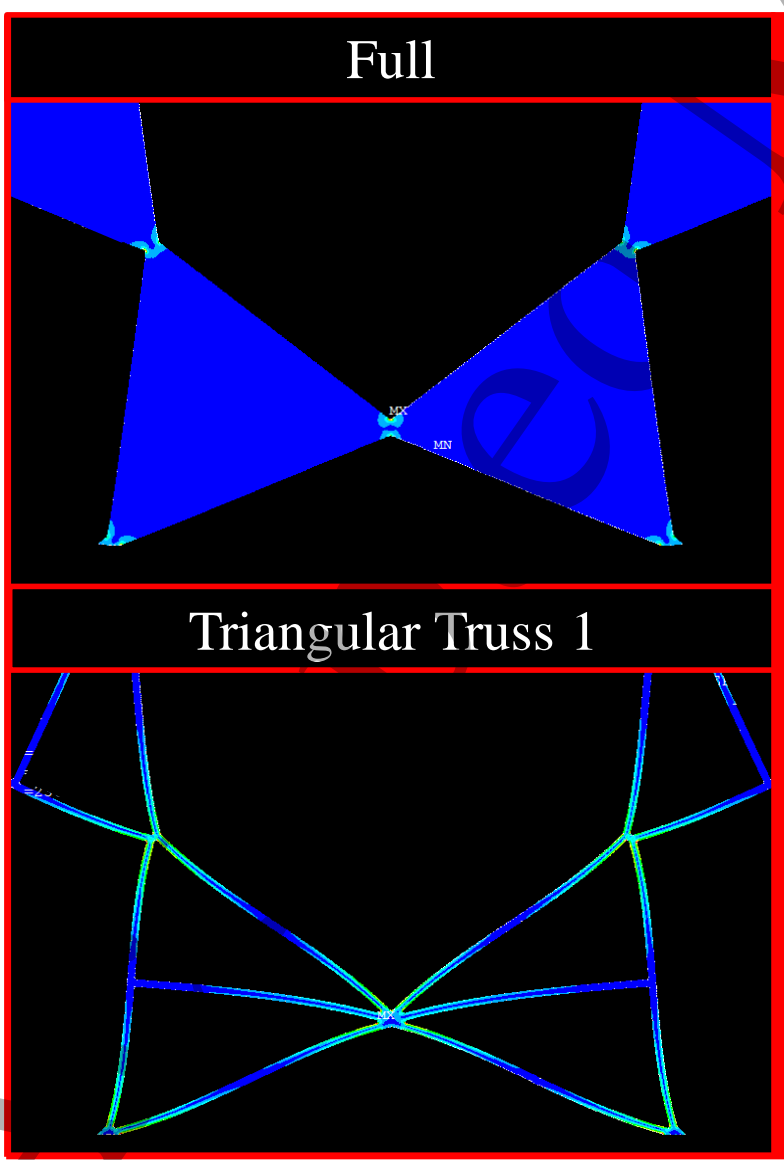

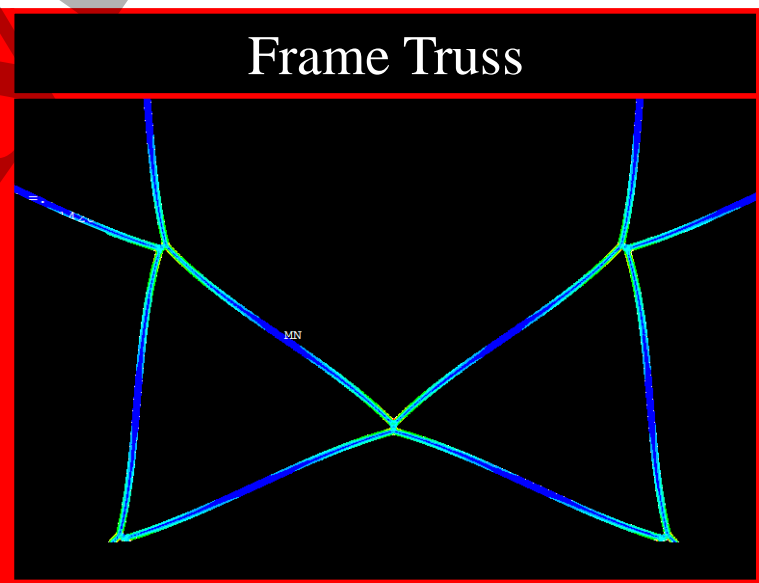

Triangular Truss 2

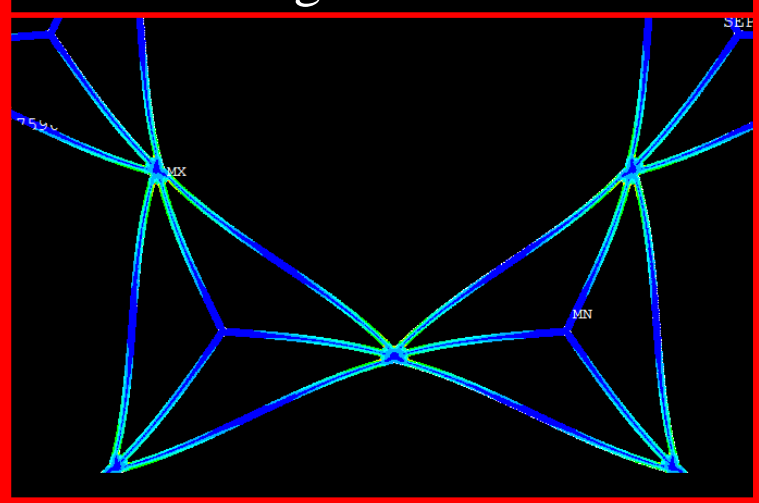


Figure 5: Images showing the equivalent Tresca stress distribution and deformed shape (with magnified displacement scaling) of the rotating triangle systems with a $\theta$ angle of $90^{\circ}$ and $t=a / 48$ for loading in the $x$ direction.

Table 2: Table presenting the absolute linear Poisson's ratios and Young's moduli values (assuming unit thickness in the $z$-direction and $E_{m a t}=1.68 \mathrm{GPa}$ ) of the rotating triangle systems with a $\theta$ angle of $90^{\circ}$ and $t$ $=a / 48$ for loading in the $x$ - and $y$-directions (systems shown in Figure 5).

\begin{tabular}{|l|c|c|c|c|}
\hline \multicolumn{1}{|c|}{ Truss Geometry } & $\boldsymbol{v}_{\boldsymbol{x} \boldsymbol{y}}$ & $\boldsymbol{v}_{\boldsymbol{y} \boldsymbol{x}}$ & $\boldsymbol{E}_{\boldsymbol{x}}(\mathbf{M P a})$ & $\boldsymbol{E}_{\boldsymbol{y}}(\mathbf{M P a})$ \\
\hline Full & -0.718 & -0.718 & 15.372 & 15.372 \\
\hline Frame Truss & -0.942 & -0.942 & 0.495 & 0.495 \\
\hline Triangular Truss 1 & -0.966 & -0.937 & 0.573 & 0.556 \\
\hline Triangular Truss 2 & -0.938 & -0.938 & 0.813 & 0.813 \\
\hline
\end{tabular}

Another important point that must be mentioned is the effect of the ligament arrangement on the isotropy of the system. The rotating triangle mechanism is known to exhibit in-plane isotropy [9] and this property appears to be retained for the full, frame truss and triangular truss 2 system as evident from Table 2. However, this appears not to be the case for the triangular truss 1 system. This is almost certainly due to the fact that the addition of a central ligament in the triangular rotating unit results in a loss of the overall hexagonal symmetry of the system, which is present for the other three configurations of this geometry.

\section{Rotating Quadrilaterals}

The plots for the mechanical properties of the three rotating quadrilateral systems investigated here (shown in Figure 6) show similar trends to the rotating triangle systems, with respect to the changes in mechanical properties upon changing rotating unit configuration, with one main exception; the frame truss systems exhibit completely different trends in comparison to the triangular truss and full geometries. In fact, regardless of the Poisson's ratio of the other rotating unit geometries, the frame truss systems consistently exhibit a highly positive Poisson's ratio. This is due to the fact that, unlike the frame rotating triangles system, the frame rotating quadrilateral systems do not possess a triangular truss arrangement within the rotating unit. This results in a loss of rigidity which in turn manifests into a loss of the auxetic rotating unit mechanism. It is evident from Figure 7, where the deformed frame rotating square system is shown, that the rotating square unit does not retain its shape and rotate as a whole but, rather, deforms in a wine-rack-like mechanism. The onset of this mechanism is characterized by highly positive Poisson's ratios, which accounts for the trends observed in the plots in Figure

6. 

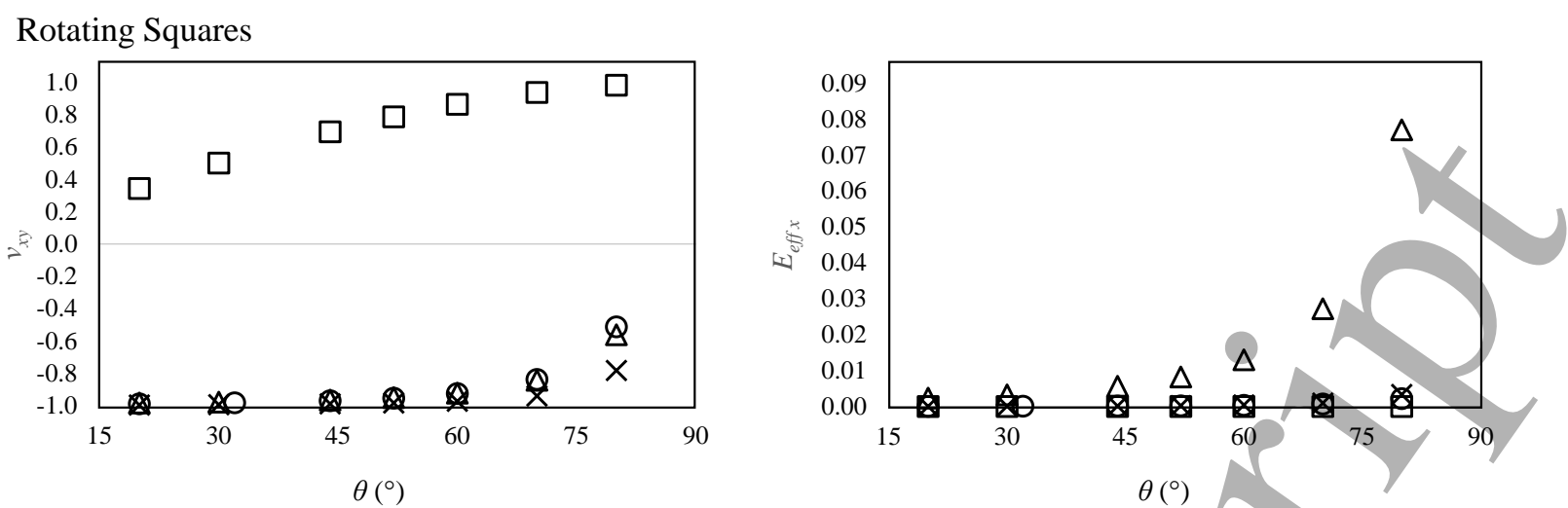

Rotating Rectangles
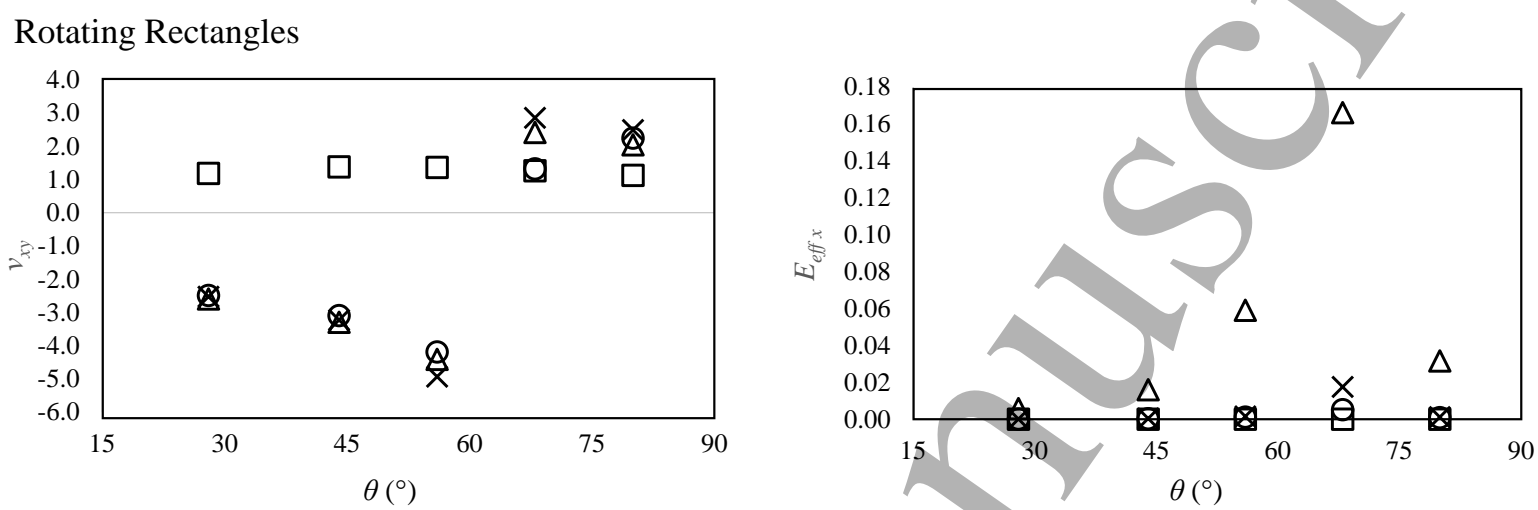

Rotating Parallelograms
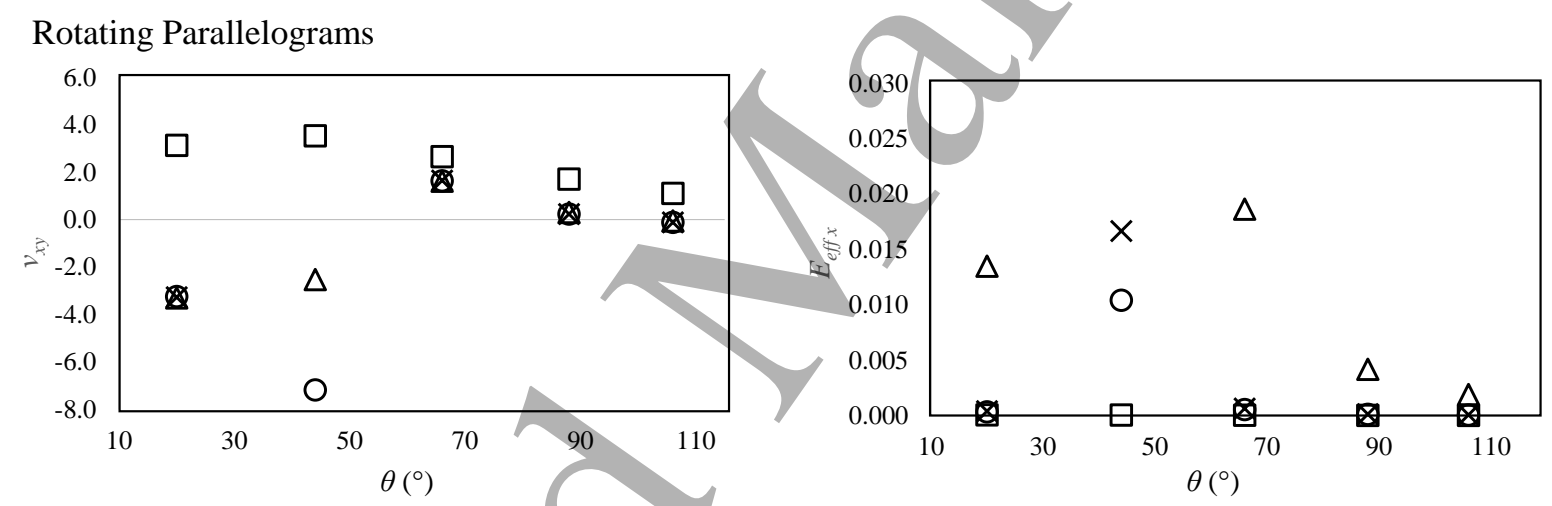

\begin{tabular}{|llllll|}
\hline Full & $\square$ & Frame & Triangular Truss 1 & $\times$ Triangular Truss 2 \\
\hline
\end{tabular}

Figure 6: Plots showing the Poisson's ratio and effective Young's moduli for the rotating square, rectangle and parallelogram systems with $t=a / 48$ loaded in the $x$-direction.

The plots in Figure 6 indicate that, on the other hand, the full and triangular truss systems all exhibit very similar Poisson's ratios, indicating that the rotating unit mechanism is still the predominant deformation mechanism for the latter systems. In the case of the rotating square systems, the strùctures with $\theta=20^{\circ}$ and $44^{\circ}$ with triangular truss and full rotating units all showed Poisson's ratio close to -1 , which is the hallmark for the rotating square mechanism. Also, for systems with a $\theta$ value of $80^{\circ}$, which is close to the fully opened conformation of $\theta=$ $90^{\circ}$, the symmetric triangular truss system (Triangular Truss 2) showed an overall smaller reduction in auxeticity in comparison to the Triangular Truss 1 and full system. Furthermore, 
as evident from Table 3, the Triangular Truss 1 system exhibits slightly anisotropic behaviour which is not observed in the Triangular Truss 2 and full systems. This behaviour is analogous to that observed for the rotating triangle systems and is similarly due to the loss of symmetry imposed on the rotating unit by the geometric configuration of the triangular truss network.
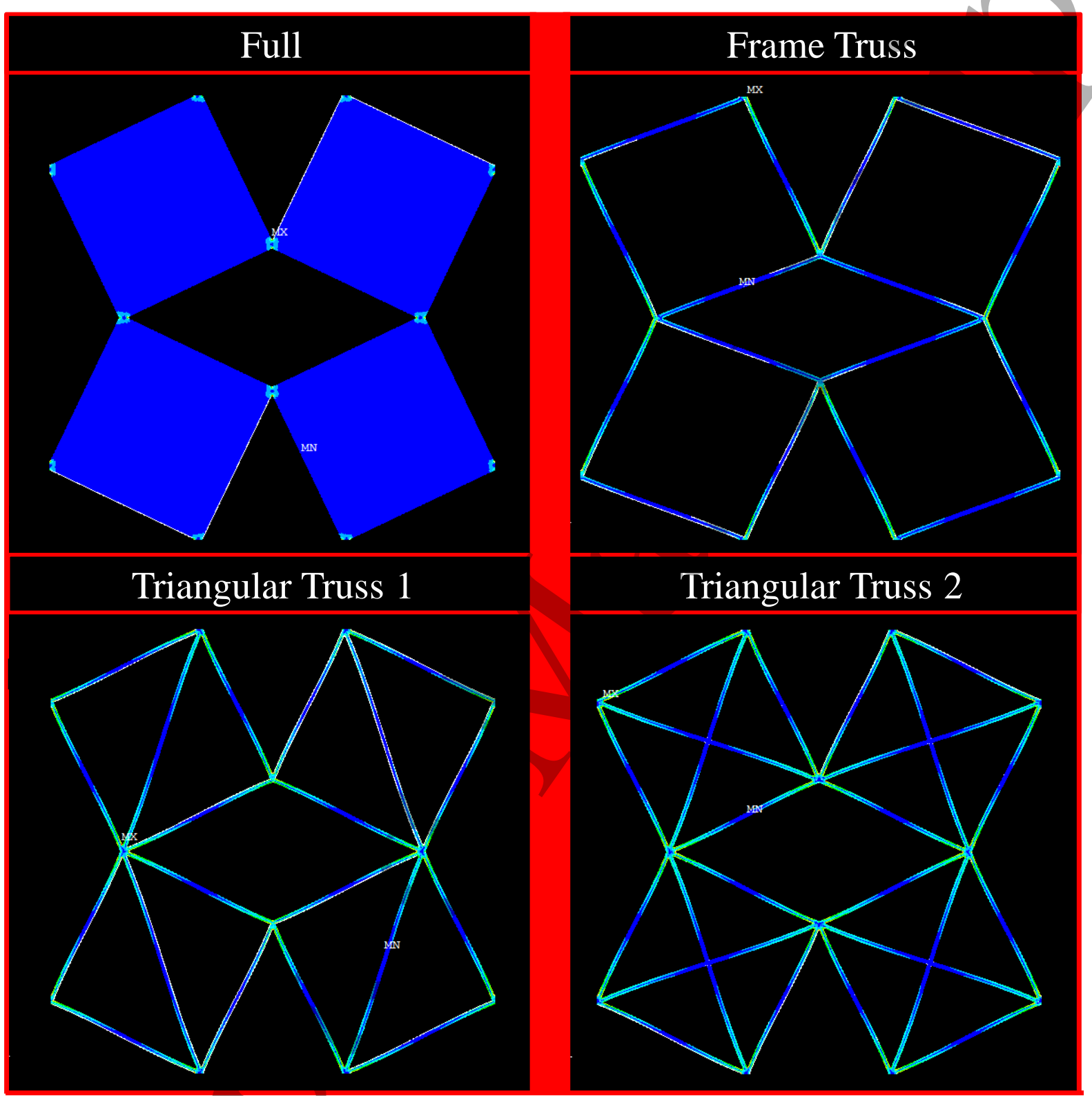

Figure 7: Images showing the equivalent Tresca stress distribution and deformed shape (with magnified displacement scaling) of the rotating square systems with a $\theta$ angle of $44^{\circ}$ and $t=a / 48$ for loading in the $x$ direction.

Table 3: Table prèsenting the absolute linear Poisson's ratios and Young's moduli values (assuming unit thickness in the $z$-direction and $E_{m a t}=1.68 \mathrm{GPa}$ ) for one set of angles of the rotating square, rectangle and parallelograms systems with $t=a / 48$ for loading in the $x$ - and $y$-directions. The chosen rotating square systems are the ones shown in Figure 7.

\begin{tabular}{|l|c|c|c|c|}
\hline \multicolumn{1}{|c|}{ Truss Geometry } & $\boldsymbol{\nu}_{x y}$ & $\boldsymbol{v}_{y x}$ & $\boldsymbol{E}_{\boldsymbol{x}}(\mathbf{M P a})$ & $\boldsymbol{E}_{\boldsymbol{y}}$ (Mpa) \\
\hline Rotating Square $\left(\boldsymbol{\theta}=\mathbf{4 4}^{\circ}\right)$ & \multicolumn{3}{|l|}{} \\
\hline Full & -0.964 & -0.964 & 9.514 & 9.514 \\
\hline
\end{tabular}




\begin{tabular}{|c|c|c|c|c|}
\hline Frame Truss & 0.695 & 0.695 & 0.0354 & 0.0354 \\
\hline Triangular Truss 1 & -0.968 & -0.980 & 0.2780 & 0.2816 \\
\hline Triangular Truss 2 & -0.988 & -0.988 & 0.3332 & 0.3332 \\
\hline \multicolumn{5}{|c|}{ Rotating Type I Rectangles $\left(\theta=44^{\circ}\right)$} \\
\hline Full & -3.296 & -0.273 & 26.964 & 2.231 \\
\hline Frame Truss & 1.384 & 0.417 & 0.0309 & 0.00932 \\
\hline Triangular Truss 1 & -3.092 & -0.298 & 0.5895 & 0.0568 \\
\hline Triangular Truss 2 & -3.229 & -0.301 & 0.7099 & 0.0661 \\
\hline \multicolumn{5}{|c|}{ Rotating Type I $\alpha$ Parallelograms $\left(\theta=66^{\circ}\right)$} \\
\hline Full & 1.620 & 0.546 & 31.320 & 10.558 \\
\hline Frame Truss & 2.655 & 0.373 & 0.04757 & 0.00668 \\
\hline Triangular Truss 1 & 1.638 & 0.593 & 0.9013 & 0.3263 \\
\hline Triangular Truss 2 & 1.653 & 0.589 & 1.0608 & 0.3784 \\
\hline
\end{tabular}

Unlike the rotating square systems, the rotating rectangle and parallelogram systems exhibit extremely high levels of anisotropy. This is an inherent property of these systems which are also well-known for their large versatility and ability to exhibit a wide range of mechanical properties ranging from highly positive Poisson's ratios to giant auxetic behaviour. This versatility is amply demonstrated in Figure 6 and Table 3, where Poisson's ratios ranging from -3.2 up to 1.6 were obtained from the full geometries of these structures. This range of Poisson's ratio was matched by the respective triangular truss counterparts of these systems, which is indicative of the retention of the rotating mechanism as the predominant deformation mechanism. On the other hand, the frame structures, while also exhibiting high levels of anisotropy, showed a positive Poisson's ratio at all times, which is the result of the wine-rack type deformation mechanism.

The Young's moduli of these systems also follow a similar trend to those of the rotating triangle systems, with the full rotating unit geometries possessing values which are several orders of magnitude higher than the truss systems. The frame systems show the lowest values, as expected, while the triangular truss systems possess more or less similar rigidities, with the Triangular Truss 2 systems exhibiting slightly higher values than their Triangular Truss 1 counterparts in all cases.

\section{Chiral Honeycombs}

The last set of structures consist of the two chiral systems; the anti-tetachiral and hexachiral honeycombs. The plots of the mechanical properties of these systems for loading in the $x$ direction are presented in Figure 8. 
Anti-Tetrachiral Honeycombs
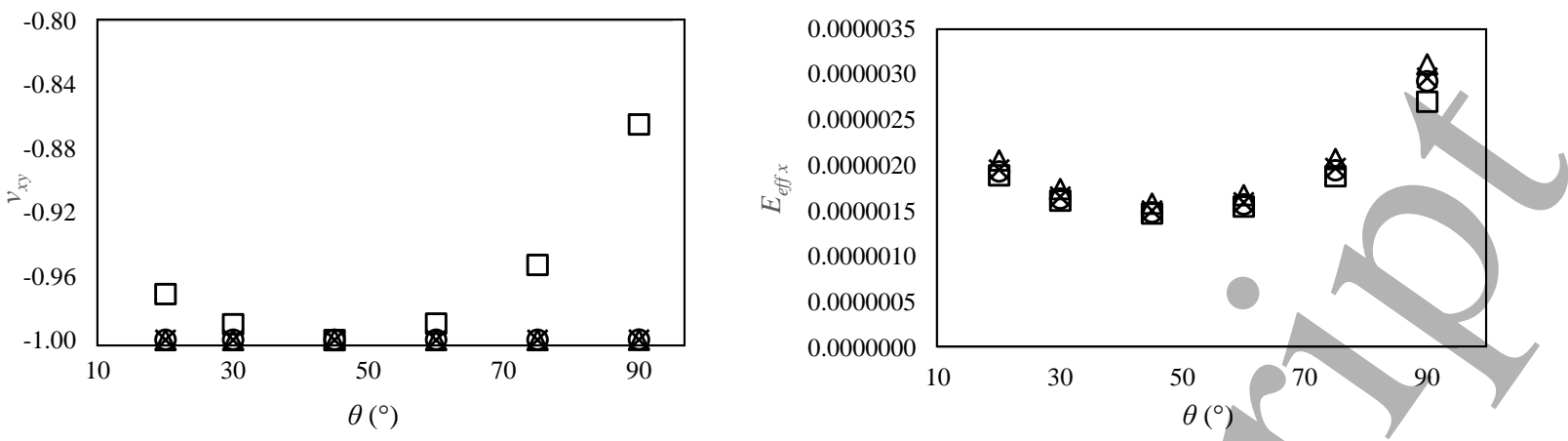

Hexachiral Honeycombs
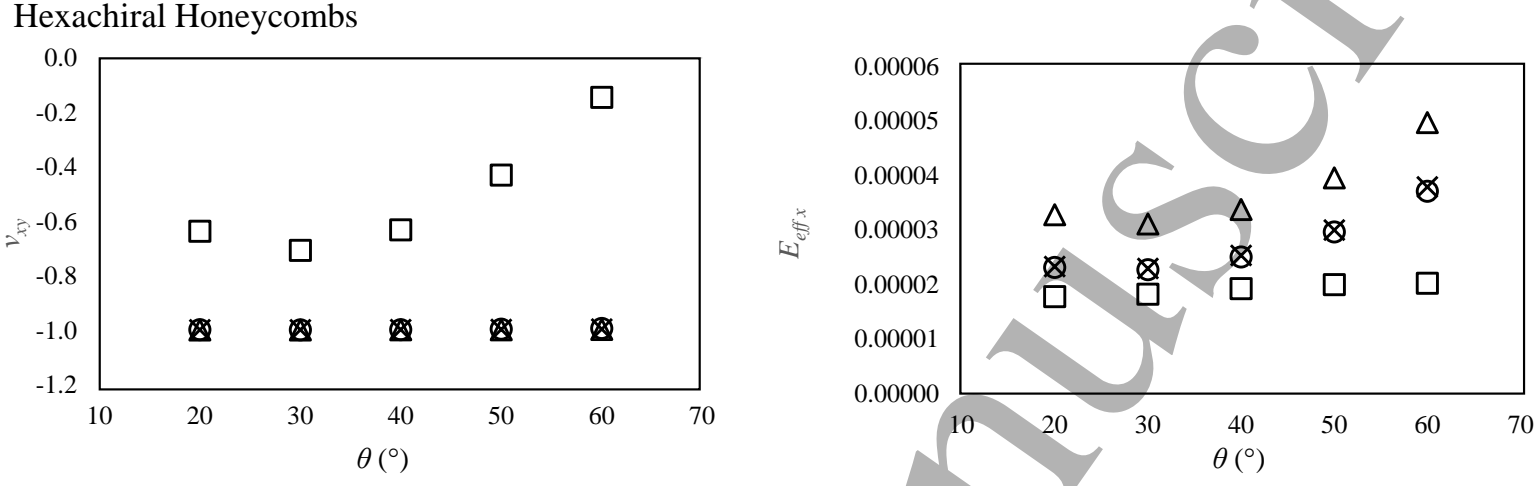

\begin{tabular}{|lllll|l|}
\hline Full & $\square$ & Frame & $\bigcirc$ & Triangular Truss 1 & $\times$ Triangular Truss 2 \\
\hline
\end{tabular}

Figure 8: Plots showing the Poisson's ratio and effective Young's moduli for the anti-tetrachiral and hexachiral systems with $t=a / 48$ loaded in the $x$-direction.

The internal geometry of the rotating unit in chiral honeycombs appears to have a less significant effect on the overall mechanical properties of the system than in the rotating rigid unit systems. In fact, the drastic changes observed for the Young's moduli of the previous structures when comparing full and truss systems were not seen for the chiral systems, with the full structures only being slightly more rigid than their truss counterparts. Moreover, although the use of a frame truss geometry resulted in a decrease of auxeticity in comparison to the full and triangular truss geometries, the large positive Poisson's ratios observed for the rotating squares, rectangles and parallelogram systems were also not evident in these systems. The antitetrachiral system, in particular, seems to be the least affected by rotating unit geometry despite having a square rotating component similar to the rotating square system, with the worst-case scenario observed here being a decrease of auxeticity from the -1 to -0.86 for the frame system with the largest $\theta$ angle. This is due to the fact that, as shown in Figure 9 below, the bulk of deformation is absorbed by the ligament connecting the rotating units together rather than the joint region, as is the case for the rotating quadrilateral and triangle systems. Since this mode of deformation is very pliant (in fact chiral honeycomb systems with long ligaments are 
characterized with a very low effective Young's modulus), the lowering of rotating unit (i.e. chiral node) rigidity by the introduction of a truss geometry does not result in a large shift of deformation mechanism as in the rotating unit systems connected via joints. This means that flexure of the connecting ligaments is still the predominant mode of deformation and hence the mechanical properties of the anti-tetrachiral systems do not change drastically upon changing the internal geometry of the rotating component (see Figure 9). However, a similar trend to that observed for the rotating square system is expected to appear should the anti-tetrachiral systems be connected by ligaments having extremely small length to thickness ratios due to the increased rigidity of the connecting ligaments.

The effect of the internal geometry of the rotating unit on the overall mechanical properties is more pronounced for the hexachiral systems for this reason. In these systems, the use of the frame geometry results in a significant decrease in auxeticity, particularly in the case of the system with $\theta=60^{\circ}$, where the Poisson's ratio varies from -1 for the full and triangular truss systems down to $c a$. -0.1 for the frame system. This drop in magnitude is primarily due to the loss of rigidity of the hexagonal rotating unit (see Figure 9), which results in deformations typical of a regular hexagonal honeycomb, which is characterized by a positive Poisson's ratio. This deformation mechanism, in conjunction with the rotation of the chiral node, results in an overall slightly negative Poisson's ratio for the entire system. 


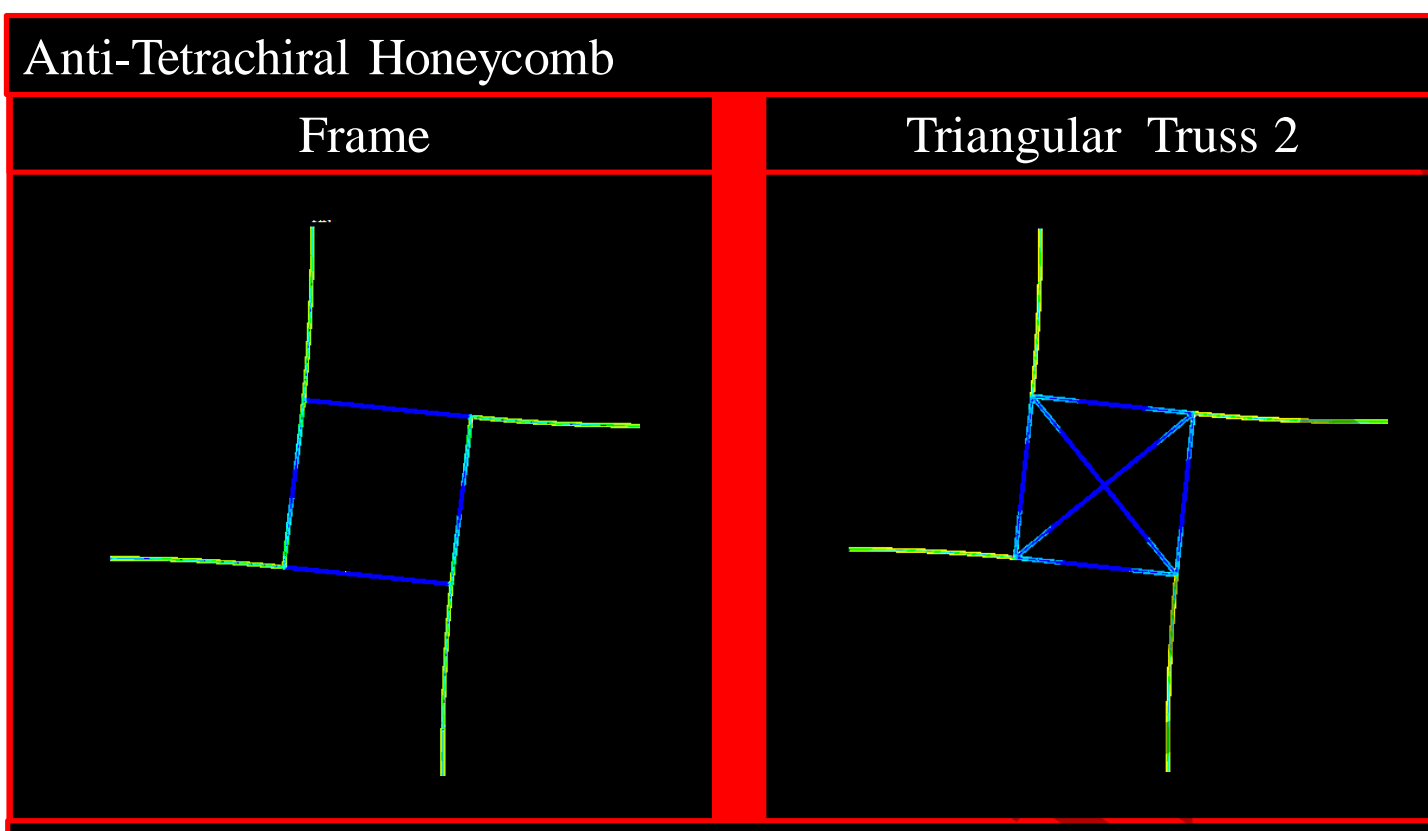

\section{Hexachiral Honeycomb}

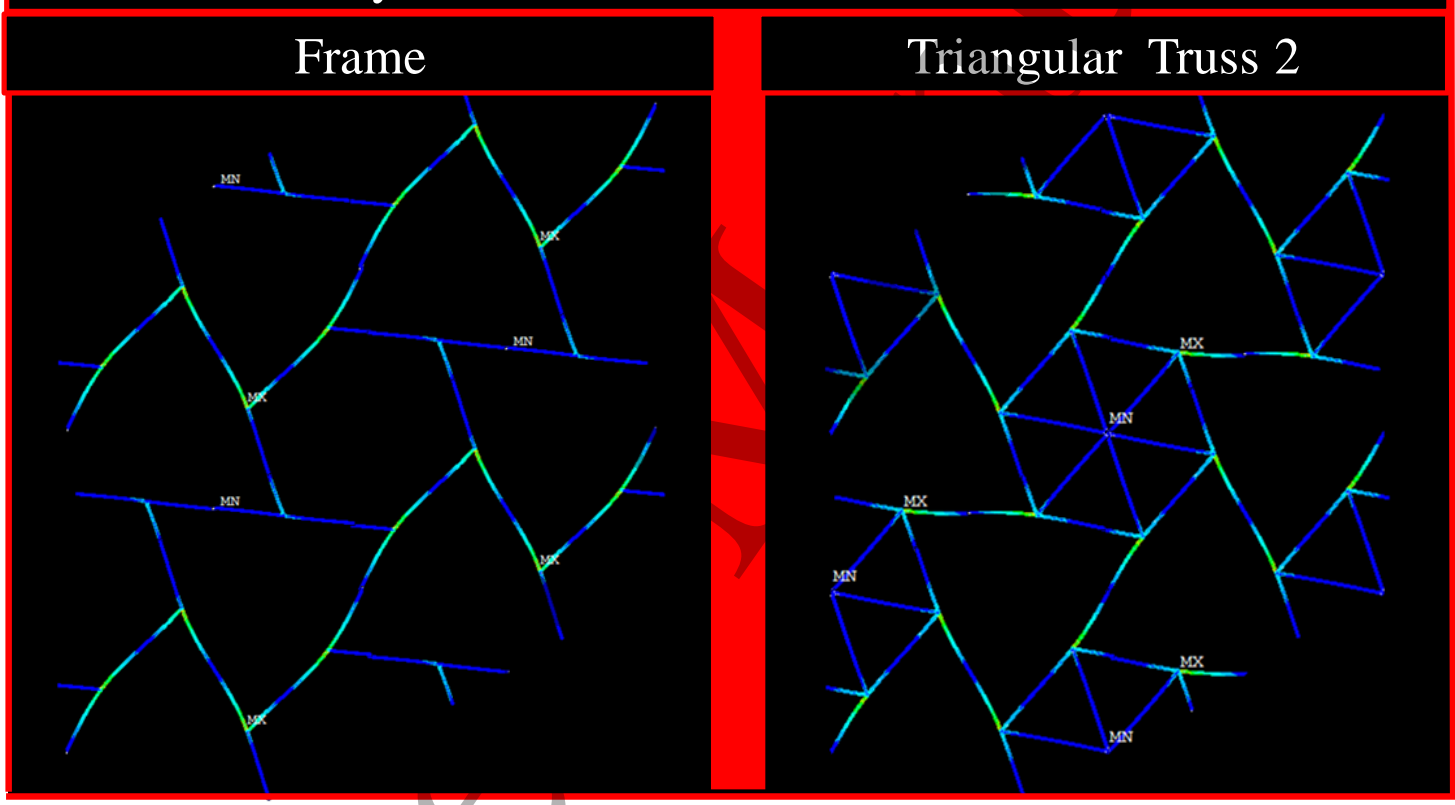

Figure 9: Relative stress intensity diagrams (Tresca stress) showing the deformation (with displacement scaling) of the anti-tetrachiral and hexachiral frame and triangular truss 2 systems with a $\theta$ angle of $90^{\circ}$ and $60^{\circ}$ respectively with $t=a / 48$ for loading in the $x$-direction. The colour blue denotes regions with minimal stress while the red regions denote maximal stress. Note, that for the sake of clarity, only a quarter of the anti-tetrachiral unit cell is shown while for the hexachiral system four adjoining unit cells are presented.

\section{Effect of Truss Thickness on Mechanical Properties}

Ligament thickness also has a significant effect on the mechanical properties of a number of systems considered here as evident from the plots shown in Figure 10. As expected, systems with thicker ligaments possess a higher effective Young's modulus than systems with thin 
ligaments regardless of the truss geometry employed. In addition, the Poisson's ratio of the truss systems generally tends towards the value of the corresponding full system as ligament thickness increases. 


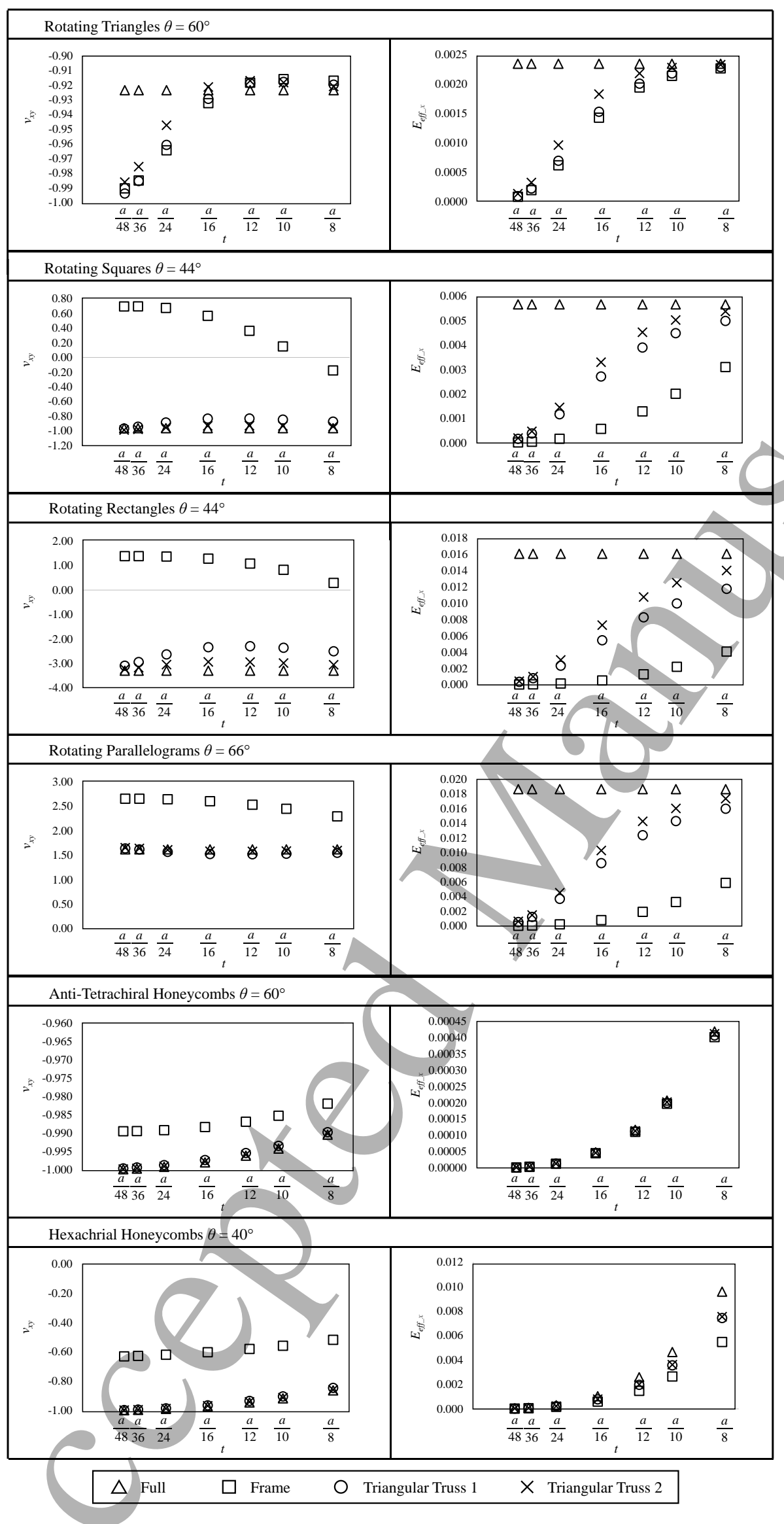

Figure 10: Plots showing how the Poisson's ratio and effective Young's moduli for all systems with one set of $\theta$ values (the intermediate value) changes with variation in $t$ when loaded in the $x$-direction. 
In the rotating 'rigid' unit systems, namely the rotating triangle and rotating quadrilateral systems, this trend is due to the fact that as the truss ligaments get thicker and, thus stiffer, they behave less like ligaments and instead of undergoing flexural deformation have a tendency to deform as rigid units. This is particularly evident in the case of the frame rotating square system with a ligament thickness of $a / 8$ (see Figure 10). This system exhibits a slightly negative Poisson's ratio of $c a$. -0.2 , while its counterparts with thinner ligaments exhibit a positive Poisson's ratio. This indicates that the rotating unit is sufficiently rigid so as to deform to some extent by rotation of the quadrilateral unit rather than primarily by internal deformations which lead to a positive Poisson's ratio.

For the chiral systems, ligament thickness has an effect on the Poisson's ratios and Young's moduli of all systems, including the full systems. This is due to the fact the fact that the main deformation mechanism of these systems is dependent on the flexural deformation of the external ligaments and therefore upon increasing the thickness of all ligaments within the system, the effective Young's modulus increases and the Poisson's ratio decreases in magnitude for all truss and full geometries. In the case of the chiral systems, there is also very little difference between the mechanical properties observed for the Triangular Truss 1, Triangular Truss 2 and Full geometries. This is particularly evident in the case of the hexachiral systems where the linear Poisson's ratios of these three configurations are almost identical for each ligament thickness value investigated.

\subsection{Experimental Results and Nonlinear Simulations}

Four rotating unit systems corresponding to each of the four structure types investigated in the linear numerical study where fabricated using 3D stereolithography printing and subjected to a small tensile load in the $y$-direction. The results of the strain $v s$ strain analysis of the central representative unit cell of the system evaluated using DIC are presented in Figure 11a while images showing the undeformed and deformed forms of these systems are presented in Figure 12. The engineering Poisson's ratio of the central repeating unit was calculated from the engineering strains of the system, which were in turn found from the measured displacements of the edges of unit cell. The displacements were obtained by point tracking of the eight edge corners which make up the repeating unit. The results of the corresponding nonlinear simulations are presented in Figure 11b, while images of the deformed structures are shown 
in Figure 12. The Poisson's ratio of the central repeating unit was measured in an identical method to that employed for the experimental results.

\section{Experimental}

a) i) ii)
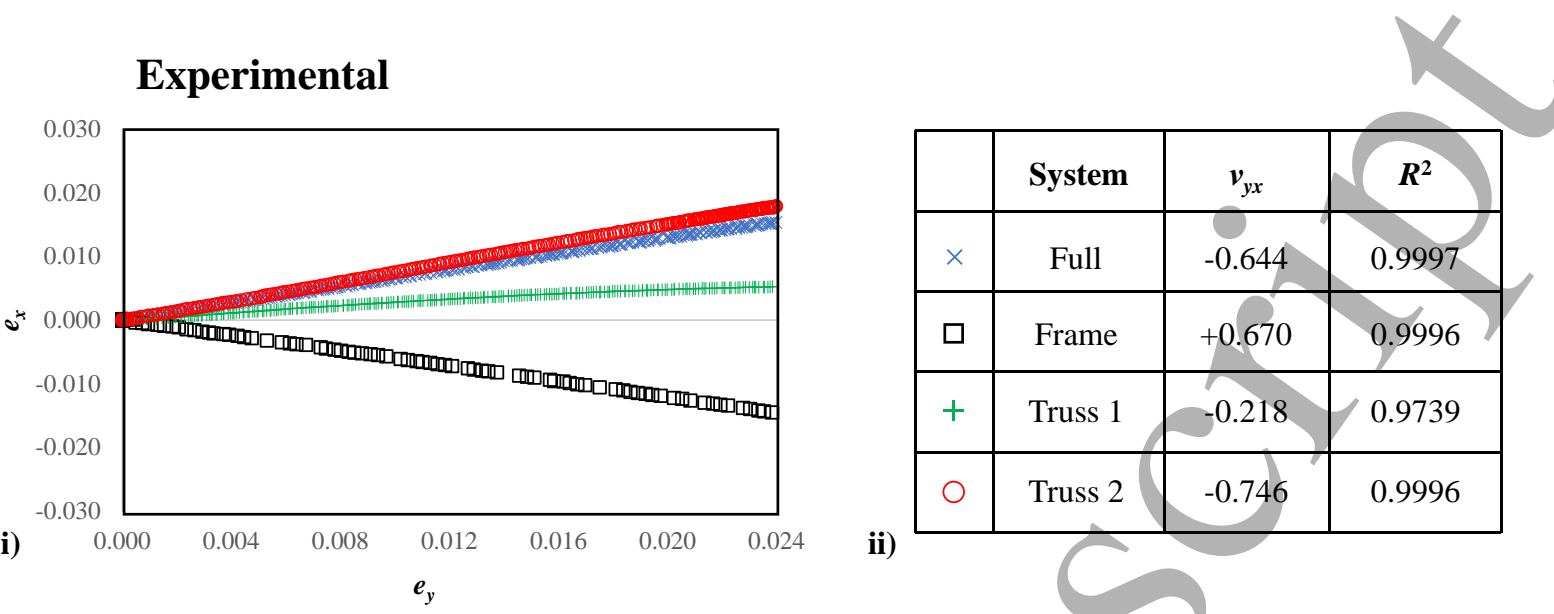

Nonlinear FE Simulations

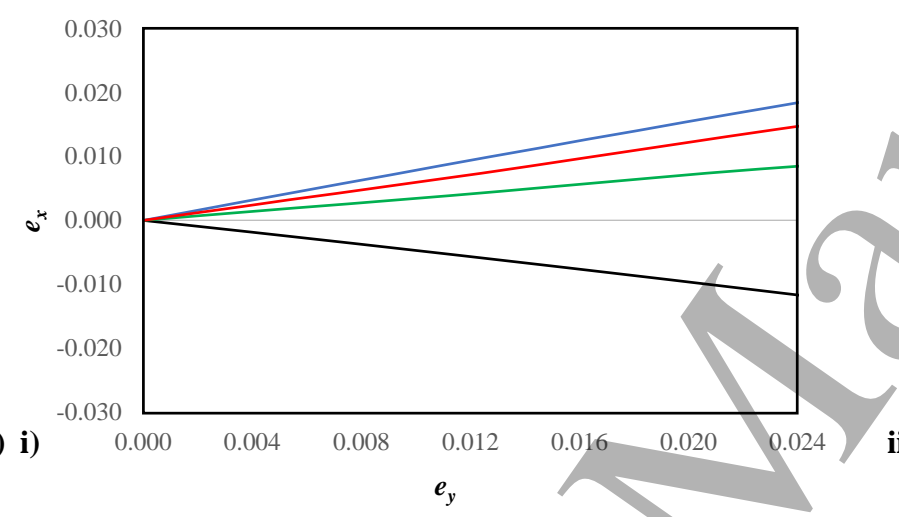

ii)

\begin{tabular}{|c|l|c|c|}
\hline & System & $\boldsymbol{v}_{\boldsymbol{y} \boldsymbol{x}}$ & $\boldsymbol{R}^{\mathbf{2}}$ \\
\hline- & Full & -0.774 & 0.9997 \\
\hline- & Frame & +0.505 & 0.9984 \\
\hline- & Truss 1 & -0.352 & 0.999 \\
\hline- & Truss 2 & -0.609 & 0.9996 \\
\hline
\end{tabular}

Figure 11: Results for the a) experimental prototypes and b) equivalent nonlinear FE simulations. i) Plots showing the engineering strain in the $y$-direction, $e_{y}$, vs the engineering strain in the $x$-direction, $e_{x}$, of the central representative unit cell over an applied tensile and ii) table showing the engineering Poisson's ratios obtained by fitting a linear model over the engineering strain data shown in the plot. 
Experimental
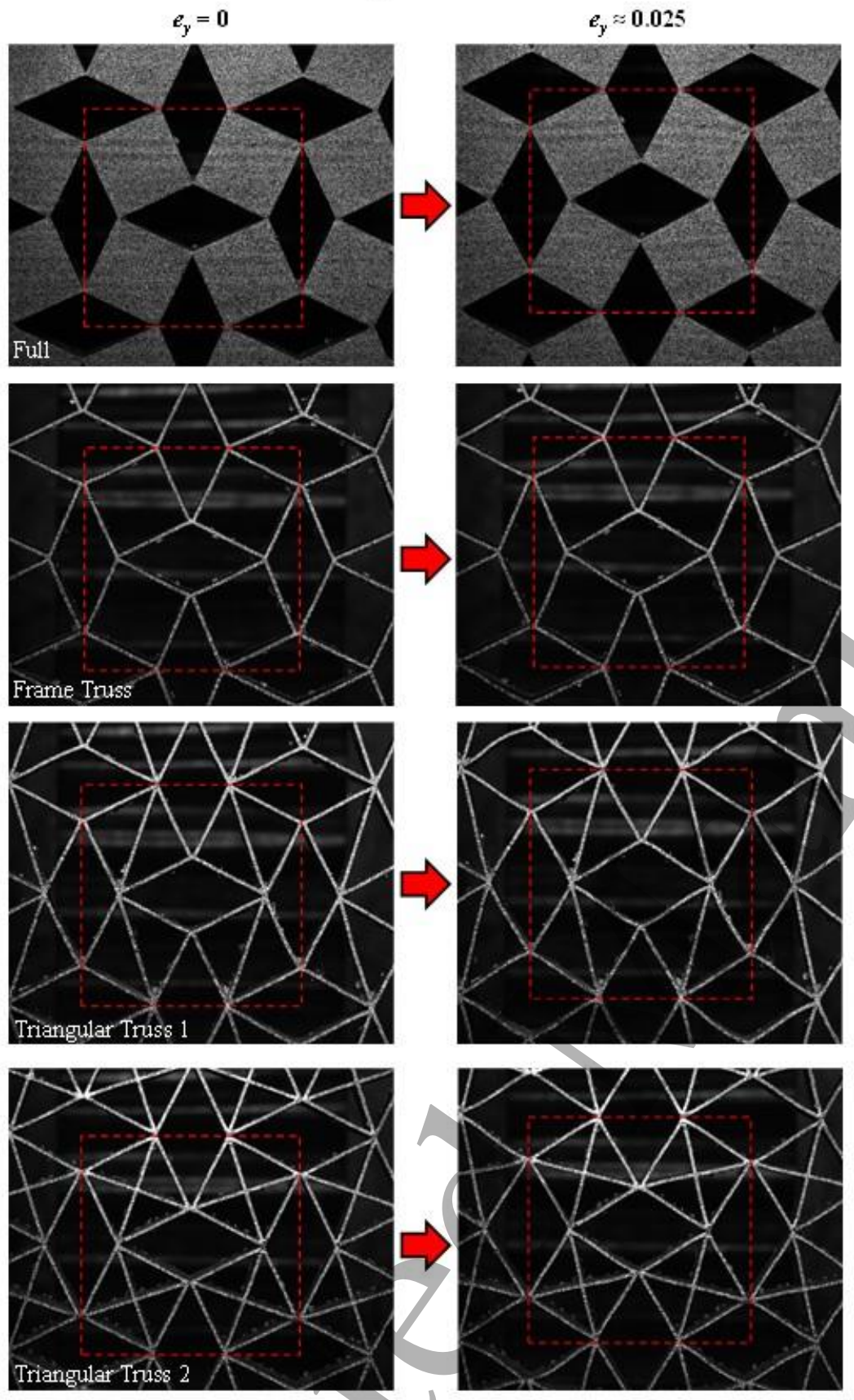

$e_{y} \approx 0.025$
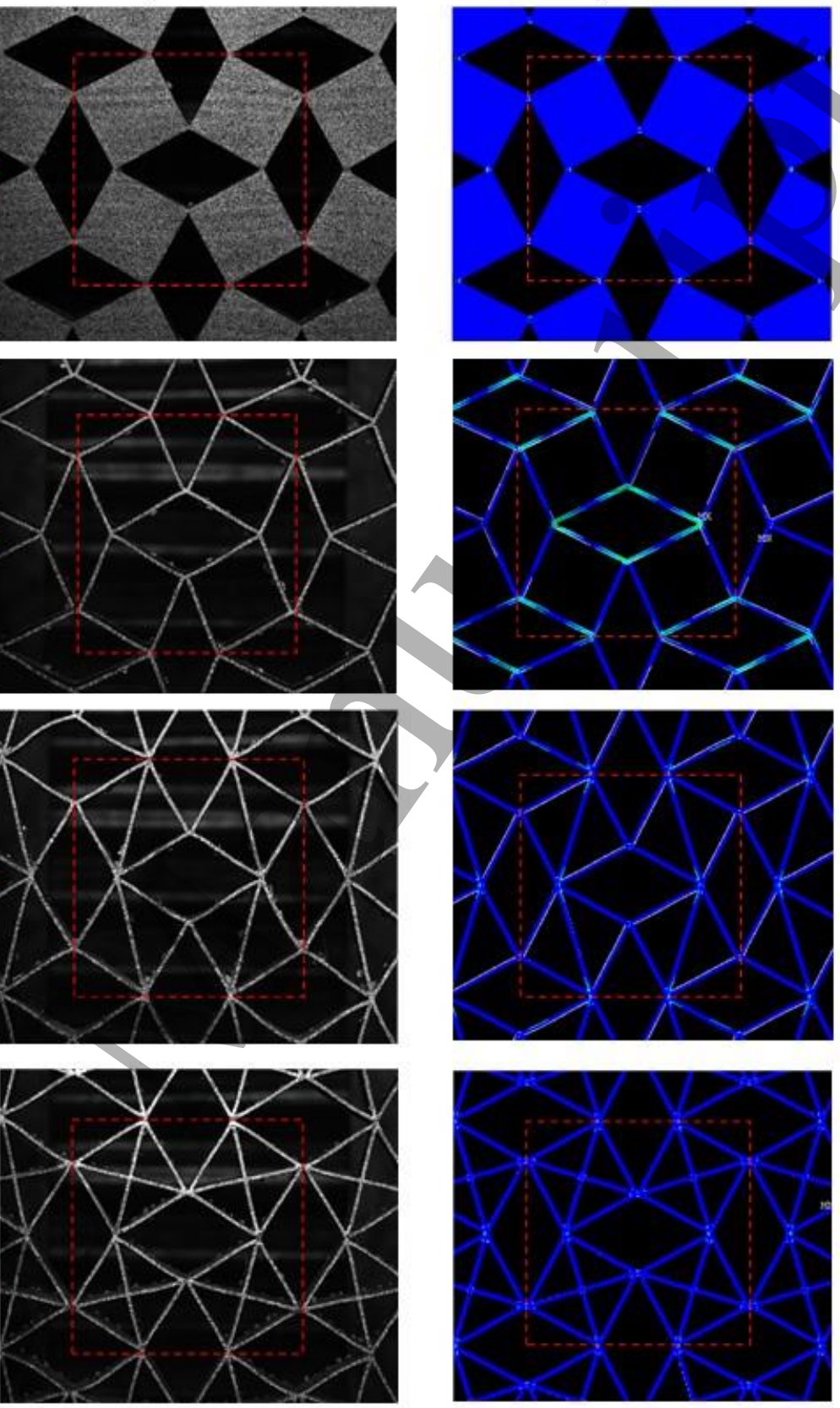

Figure 12: Images showing the undeformed and deformed states $\left(e_{y} \approx 0.025 \%\right)$ of the central representative unit cell of each system for the experimental and nonlinear FE simulations.

As one may observe from the plots in Figure 11, the small strain deformation behaviour of each system changes in a mainly linear manner and thus it is possible to obtain the linear Poisson's ratio for strains less than $2.5 \%$. The Poisson's ratios obtained from the experimental tests and the equivalent nonlinear simulations are extremely similar and as predicted earlier by the linear numerical simulations, the Frame system showed a positive Poisson's ratio, while the other systems each possess a negative Poisson's ratio. The deformation profile of both sets of systems are also nearly identical. The magnitudes of the Poisson's ratios are lower than those 
obtained from the equivalent periodic linear simulations in all cases $($ Full $=-0.958$, Frame $=$ 0.715, Triangular Truss $1=-0.912$ and Triangular Truss $2=-0.939$ ); this was to be expected since the experimental and nonlinear FE models contained only $3 \times 3$ repeating units (due to $3 \mathrm{D}$ printing constraints), which are not sufficient to completely eliminate boundary effects. This edge effect has been previously observed in a range of other metamaterial geometries $[37,40]$ and the discrepancy between the periodic and experimental models (as well as the non-periodic FE simulations) can be reduced by using a larger number of representative units in the latter systems.

The experimental Full and Triangular Truss 2 systems exhibited very similar Poisson's ratios of -0.64 and -0.75 respectively, while the Triangular Truss 1 showed a significant decrease in magnitude in comparison, with a Poisson's ratio of -0.22 being observed for this system. While the similarity between the auxeticity of the Full and Triangular Truss $2 /$ system was expected, in view of the numerical simulation results presented in the previous sections, the Poisson's ratio value observed for the Triangular Truss 1 system was surprising. However, the reason for this discrepancy can be explained by observing the deformed state of this system. As is evident from Figure 12, the square 'rotating units' of the Triangular Truss 1 system are observed to distort significantly at $2.5 \%$ strain in comparison to those in the Triangular Truss 2 system, although not to the same extent as the Frame Truss structure. This behaviour, which was also observed, albeit to a much lesser extent, in the linear FE simulations, appears to indicate that the additional reinforcement and retention of symmetry of the Triangular Truss 2 system is necessary over larger strains in order for the rotating unit to retain its original polygonal shape. Moreover, the significant amount of flexural deformation observed in both triangular truss systems suggests that at higher strains the global deformation and mechanical properties of these systems are expected to differ significantly from those of their full counterpart, which has no ligaments. This hypothesis is based on the well-known propensity of the effective Young's modulus of rotating rigid unit mechanisms to increase as the systems approach their fully-opened state and therefore, one would expect alternative deformation mechanism to play a more significant role in the global deformation of these systems at higher strains. In the case of the full systems, the alternative mechanism involved is typically stretching of the 'joint' regions connecting the rotating units [40-42], however in the case of the truss networks it is envisaged that this could take the form of flexural deformation of ligaments. Further studies on the high strain behaviour of these systems are required in order to confirm this point. 


\section{$\underline{3.3 \text { Overall Discussion }}$}

The results obtained from both the FE simulations and experimental tests show that the introduction of a triangular truss system in place of rotating units made up of a solid 'filled' block of material is a viable route towards the design of lightweight ligament-based auxetic systems. The systems investigated here involved a wide range of auxetic metamaterials which fall under the classification of chiral and rotating 'rigid' unit systems and range from symmetric isotropic geometries, namely hexachiral honeycombs, rotating triangles and squares, to highly anisotropic ones, i.e. rotating rectangle and parallelogram systems. In each case, it was observed that the on-axis small-strain Poisson's ratios of the triangular truss systems were comparable to those of their full counterparts, indicating that the introduction of a Level 0 triangular truss-based hierarchical element does not result in a significant loss of auxeticity. Furthermore, as shown in the plot in Figure 13, the proposed truss-based systems can be constructed using at least $80 \%$ less material for systems with a ligament thickness of $a / 48$ (i.e. the systems which mechanical properties are presented in Figures 4, 6 and 8) in comparison to the full systems. Obviously, for systems with thicker ligaments, the relative surface coverage, and hence the mass, of the truss systems is higher. In each case, as expected, the frame truss systems possessed the least amount of material, however as shown previously, this comes at the cost of a considerable reduction or loss of auxeticity in all cases except for the rotating triangle systems. However, this is not the case for the triangular truss systems which are composed of a comparable amount of material.

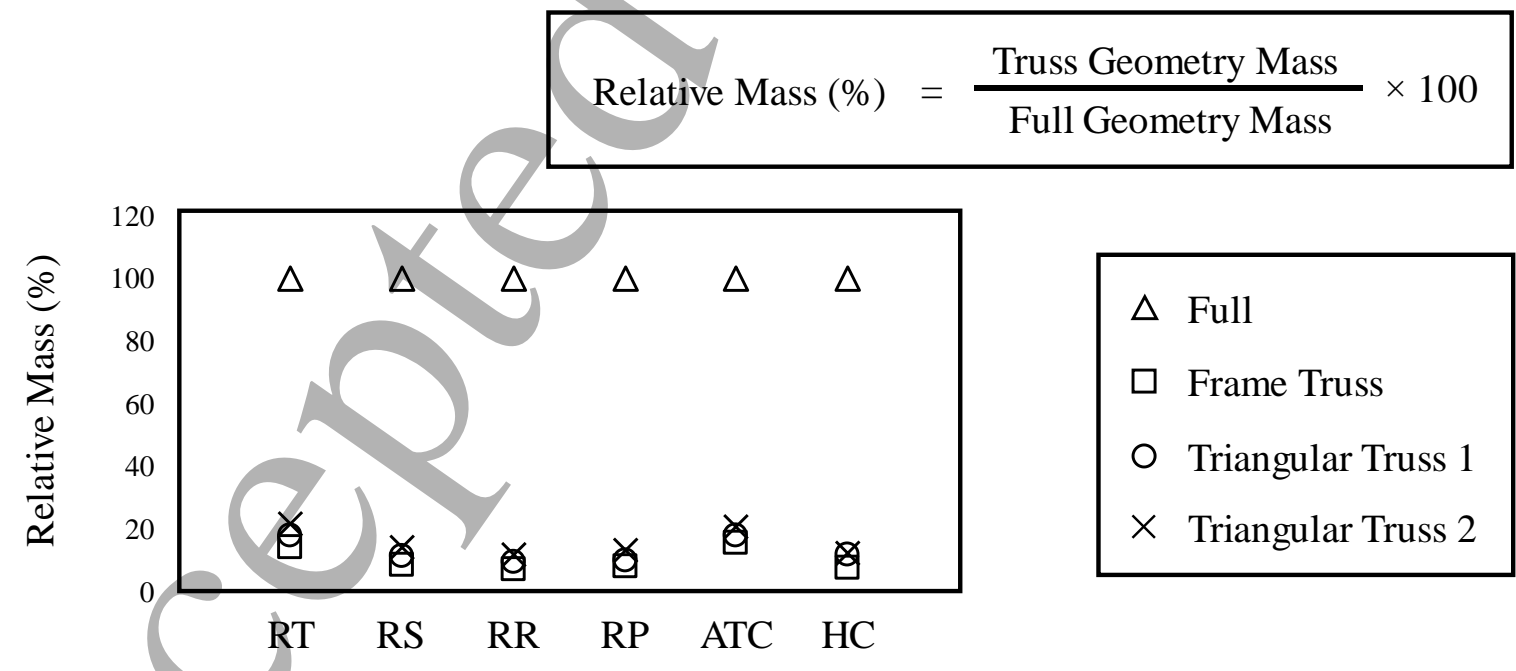

Figure 13: Plot showing the relative mass of each truss configuration (in terms of surface area coverage) for every metamaterial system in comparison with that of the corresponding full version. The points represent systems with a truss thickness of $a / 48$ and are given for rotating triangle (RT), rotating square (RS), rotating rectangle (RR), rotating parallelogram (RP) systems, anti-tetrachiral (ATC) and hexachiral (HC) honeycombs. 
One of the main consequences of introducing a hierarchical truss-based configuration into rotating unit metamaterials is a significant decrease in the stiffness of the system. This is particularly significant in the case of systems made from extremely thin ligaments where a drop of two orders of magnitude may be observed in the cases of rotating 'rigid' unit-type systems. As mentioned previously, this is mainly a result of the change in deformation mechanism from rotation-dominated deformation to concurrent rotation and flexure of ligaments making up the rotating unit. This, in turn, results in a less localized concentration of stresses within the geometry (as shown in Figures 5 and 7), with the deformation response to loading of the system being dispersed throughout the flexing ligaments and the 'joint' regions. While this causes a significant drop in structural stiffness, this effect is also expected to enhance the deformability and fatigue performance of these systems for a given applied strain. This factor has been observed in previous studies for optimization of the 'joint' regions of rotating unit auxetics, where a decreased concentration of stress at these regions results in a lower effective Young's modulus and increased strain tolerance $[33,43]$.

In order to analyze closely the trade-off between mass and resultant stiffness of the metamaterial geometries studied here, we conducted an additional investigation on the truss systems which possess nearly identical Poisson's ratios to their full counterparts. This analysis was quantified in terms of the resultant apparent Young's modulus, $E^{*}$, to apparent density, $\rho^{*}$, ratio of the full and truss equivalents of these systems. This relationship may be determined by the equation below:

$$
\frac{E^{*}}{\rho^{*}}=\frac{E_{e f f}}{V_{f}} \frac{E_{m a t}}{\rho_{m a t}}
$$

where $E_{\text {eff }}$ represents the effective Young's modulus, $V_{f}$, the volume fraction of the material and $E_{m a t}$ and $\rho_{m a t}$, the material Young's modulus and density respectively. The volume fraction is defined as the ratio of material volume in a repeating unit divided by the volume of the entire repeating unit including void volume. The terms $E_{\text {eff }} / V_{f}$ determine the structural/geometric constant of the $E^{*} / \rho^{*}$ relationship for a given geometry while $E_{\text {mat }} / \rho_{\text {mat }}$ define the material constant (which is typically found from Ashby plots [44]). In Figure 14, a comparison of the geometric ratio of Full, Triangular Truss 1 and Triangular Truss 2 systems for rotating triangles, squares and hexachiral honeycombs is presented with a thickness of $a / 48$ and $a / 8$ is presented (frame structures were excluded due to the fact that they do not retain the same 
Poisson's ratios in the case of rotating squares and hexachiral honeycombs). As one may observe from these plots, the trends observed for the systems with thick ligaments differ significantly from those observed for the thin ligament structures. While for systems with thick ligaments, the truss geometries exhibit larger $E_{\text {eff }} / V_{f}$ ratios in comparison to their full counterparts, the opposite is observed for the systems with thin ligaments, where the highest ratios were observed for the full systems (except in the case of the hexachiral system where comparable values were observed for all three types). This indicates that the introduction of the hierarchical triangular truss geometry within the rotating unit also allows one to tailor the stiffness/density ratio of the metamaterial structure whilst retaining the same Poisson's ratio simply by changing the thickness of the ligaments and that one can decrease or increase this ratio with respect to the full system by design.

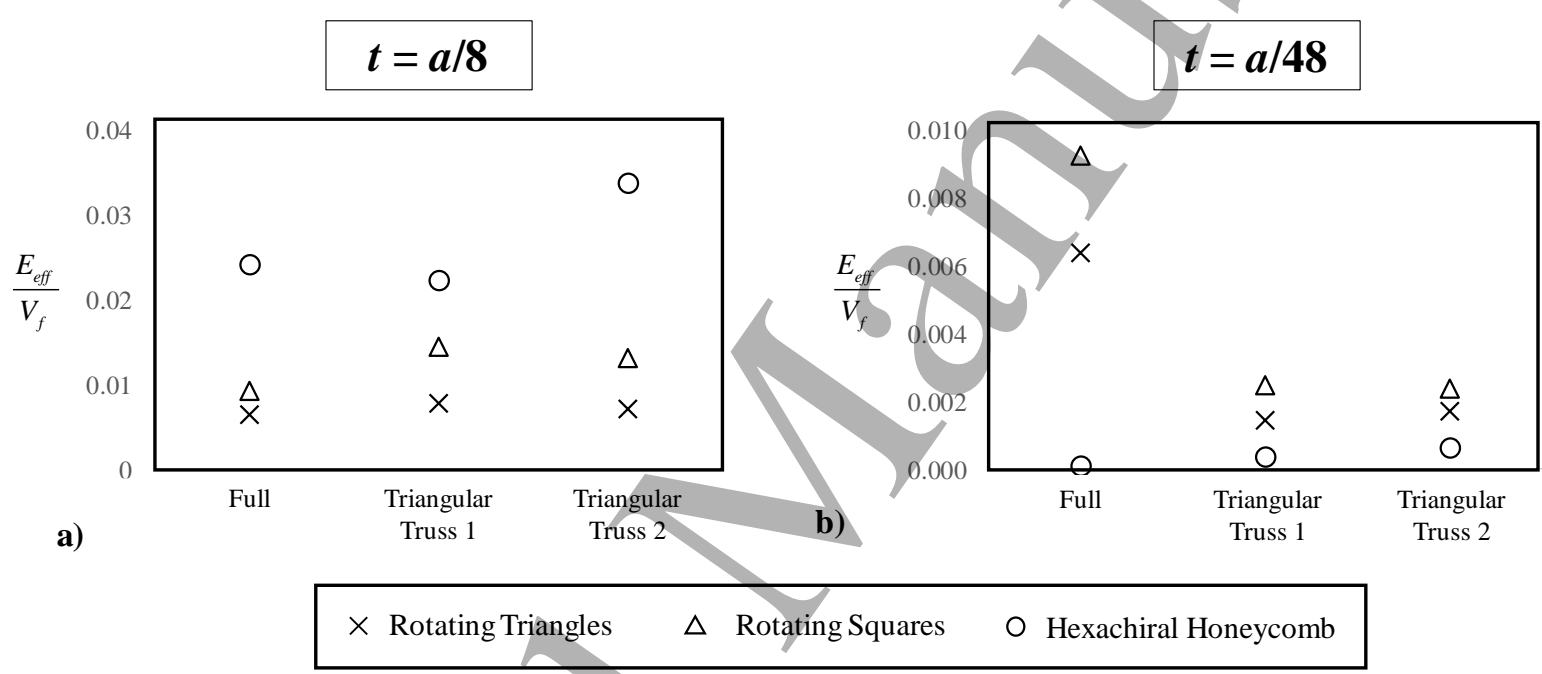

Figure 14: Plot showing changes in $E_{\text {eff }} / V_{f}$ ratios observed for a set of rotating triangles $\left(\theta=60^{\circ}\right)$, rotating squares $\left(\theta=44^{\circ}\right)$ and hexachiral honeycombs $\left(\theta=40^{\circ}\right)$ with ligament thickness values of a) $t=a / 8$ and $\left.b\right) t$ $=a / 48$ upon changing the geometry of the rotating unit (i.e. full, triangular truss 1 and triangular truss 2 )

At this point it is important to highlight the fact that the analysis conducted here was concerned primarily with the small-strain deformation and mechanical properties of these systems. While the findings obtained in this work indicate that the Poisson's ratio of a rotating system may be retained through the introduction of a hierarchical triangular truss geometry, further studies are required in order to confirm whether this is the case at higher strains, with deformations such as buckling of ligaments expected to play a more prominent role under these conditions. In addition, another point of interest is that the fact that many of the truss systems have a very low effective Young's modulus in comparison to their full counterparts. While this factor provides additional versatility to the design of these systems by allowing one to customize the stiffness 
of these metamaterials whilst retaining the Poisson's ratio, it would also be of interest to analyse under what geometric conditions (i.e. ligament thickness, triangular truss system, etc.) can a hierarchical system possess an identical effective Young's modulus with respect to its full counterpart. Further studies, including geometry optimization studies and a detailed high strain analysis, need to be conducted in order to analyse these factors and understand the full potential of these systems.

Before concluding, it is necessary to mention the advantages and potential applicability of the systems proposed here. Lightweight auxetic metamaterials have been proposed for a variety of uses including cores in sandwich systems $[45,46]$, damping systems in airfoils $[47,48]$ and as deployable structures $[49,50]$. Most of these applications incorporate the use of ligament-based systems such as re-entrant hexagonal honeycombs and chiral systems and thus the systems proposed here could potentially be used as an additional option. Full rotating 'rigid' unit auxetics are in general known to possess higher effective Young's moduli in comparison to chiral and re-entrant geometries and hence, if the correct balance of geometric parameters is maintained, the introduction of truss-geometries could provide an alternative route for the design of light metamaterials based on these mechanisms which still retain a considerable level of stiffness. In addition, the truss-based systems proposed here could also be utilised in applications where a high degree of porosity is required such as in biomedical scaffolds and stents. At this point it is also imperative to mention that while in this work truss-based configurations were applied to a wide range of $2 \mathrm{D}$ auxetic systems only, the same concept could also be potentially extended to 3D rotating unit auxetic systems where the reduction in material volume used and increase in porosity is expected to be considerably greater than in the cases proposed here. An analysis of such 3D systems along with an investigation on the high strain properties of truss-based rotating unit systems is the next step towards the eventual implementation of such systems in real-life applications.

\section{Conclusion}

In this work a wide range of rotating unit auxetic systems were designed using three types of hierarchical truss-based geometries and evaluated using a numerical FE approach and experimental testing on 3D printed prototypes. The results obtained show that while the use of frame-based geometries is inadmissible in most cases since it leads to a drastic change in deformation mechanism and a loss of auxetic behaviour, the Poisson's ratios of systems 
possessing triangular truss-based configurations are almost identical to those of their full counterparts, making them ideal candidates for the design of lightweight auxetic systems. In addition, most of the triangular truss systems were shown to be less stiff than corresponding full systems. It is envisaged that such systems could be utilised in the future in applications requiring lightweight highly porous metamaterial structures.

\section{$\underline{\text { References }}$}

[1] Evans KE, Nkansah MA, Hutchinson IJ, Rogers JA. Molecular network design. Nature $1991 ; 353: 124$.

[2] Lakes R. Foam Structures with a Negative Poisson's Ratio. Science (80- ) 1987;235:1038-40. doi:10.1126/science.235.4792.1038.

[3] Wojciechowski KW, Branka AC. Negative Poisson ratio in a two-dimensional "“isotropic"” solid. Phys Rev A 1989;40:7222-5.

[4] Gibson LJ, Ashby MF, Schajer GS, Robertson CI. The Mechanics of Two Dimensional Cellular Materials. Proc R Soc A Math Phys Eng Sci 1982;382:25-42.

[5] Evans BKE, Alderson A. Auxetic Materials : Functional Materials and Structures from Lateral Thinking! Adv Mater 2000:617-28.

[6] Scarpa F, Giacomin JA, Bezazi A, Bullough WA. Dynamic behavior and damping capacity of auxetic foam pads. Smart Mater Struct 2006:61690T. doi:10.1117/12.658453.

[7] Bacigalupo A, Lepidi M, Gnecco G, Gambarotta L. Optimal design of auxetic hexachiral metamaterials with local resonators. Smart Mater Struct 2016;25:1-41. doi:10.1088/0964-1726/25/5/054009.

[8] Grima JN, Evans KE. Auxetic behavior from rotating squares. J Mater Sci Lett 2000;19:1563-5.

[9] Grima JN, Evans KE. Auxetic behaviour from rotating triangles. J Mater Sci 2006;41:3193-6.

[10] Grima JN, Chetcuti E, Manicaro E, Attard D, Gatt R, Evans KE. On the auxetic 
properties of generic rotating rigid triangles On the auxetic properties of generic rotating 2012. doi:10.1098/rspa.2011.0273.

[11] Grima JN, Gatt R, Alderson A, Evans KE. On the auxetic properties of 'rotating rectangles' with different connectivity. J Phys Soc Japan 2005;74:2866-7.

[12] Attard D, Manicaro E, Grima JN. On rotating rigid parallelograms and their potential for exhibiting auxetic behaviour. Phys Status Solidi B 2009;2044:2033-44. doi:10.1002/pssb.200982034.

[13] Grima, Joseph N., Farrugia P-S, Gatt R, Attard D. On the auxetic properties of rotating rhombi and parallelograms: A preliminary investigation. Phys Status Solidi 2008;245:521-9.

[14] Attard D, Grima JN. A three-dimensional rotating rigid units network exhibiting negative Poisson's ratios. Phys Status Solidi 2012;249:1330-8.

[15] Prall D, Lakes RS. Properties of a chiral honeycomb with a Poisson's ratio of -1. Int J Mech Sci 1997;39:305-14.

[16] Wojciechowski KW. Two-dimensional isotropic system with a negative poisson ratio. Phys Lett A 1989;137:60-4. doi:10.1016/0375-9601(89)90971-7.

[17] Sigmund O, Torquato S. Design of smart composite materials using topology optimization. Smart Mater Struct 1999;8:365-79. doi:10.1088/0964-1726/8/3/308.

[18] Dudek KK, Gatt R, Wojciechowski/KW, Grima JN. Self-induced global rotation of chiral and other mechanical metamaterials. Int J Solids Struct 2020;191:212-9.

[19] Wu W, Hu W, Qian G, Liao H, Xu X, Berto F. Mechanical design and multifunctional applications of chiral mechanical metamaterials: A review. Mater Des 2019;180:107950.

[20] Spadoni A, Ruzzene M, Numerical and experimental analysis of the static compliance of chiral truss-core airfoils. J Mech Mater Struct 2007;2:965-81.

[21] $\mathrm{Wu}$ L, Li B, Zhou J. Isotropic negative thermal expansion metamaterials. ACS Appl Mater Interfaces 2016;8:17721-7.

[22] Ebrahimi H, Mousanezhad D, Nayeb-hashemi H, Norato J, Vaziri A. 3D cellular metamaterials with planar anti-chiral topology. Mater Des 2018;145:226-31. 
[23] Gatt R, Mizzi L, Azzopardi JI, Azzopardi KM, Attard D, Casha A, et al. Hierarchical Auxetic Mechanical Metamaterials. Sci Rep 2015;5:1-6. doi:10.1038/srep08395.

[24] Dudek KK, Gatt R, Mizzi L, Dudek MR, Attard D, Evans KE, et al. On the dynamics and control of mechanical properties of hierarchical rotating rigid unit auxetics. Sci Rep 2017;7:65-9. doi:10.1038/srep46529.

[25] Cho Y, Shin J, Costa A, Ann T, Kunin V, Li J, et al. Engineering the shape and structure of materials by fractal cut. Proc Natl Acad Sci 2014;111:17390-5. doi:10.1073/pnas.1417276111.

[26] Li D, Yin J, Dong L, Lakes RS. Numerical analysis on mechanical behaviours of hierarchical cellular structures with negative Poisson's ratio. Smart Mater Struct 2016;26:025014.

[27] Rayneau-Kirkhope D. Stiff auxetics: Hierarchy as a route to stiff, strong lattice based auxetic meta-materials. Sci Rep 2018;8:12437.

[28] Dragoni E, Ciace VA. Mechanical design and modelling of lightweight additively manufactured lattice structures evolved from regular three-dimensional tessellations. Proc Inst Mech Eng Part C J Mech Eng Sci 2019. doi:10.1177/0954406219885959.

[29] Zheng X, Smith W, Jackson J, Moran B, Cui H, Chen D, et al. Multiscale metallic metamaterials. Nat Mater 2016;15:1100-7. doi:10.1038/NMAT4694.

[30] Turco E, Giorgio I, Misra A, Isola F. King post truss as a motif for internal structure of (meta) material with controlled elastic properties. R Soc Open Sci 2017;4:171153. doi:10.1007/s00033-015-0556-4.

[31] Lakes R. Materials with structural hierarchy. Nature 1993;361:511-5.

[32] Meza LR, Zelhofer AJ, Clarke N, Mateos AJ, Kochmann DM, Greer JR. Resilient 3D hierarchical architected metamaterials. Proc Natl Acad Sci 2015;112:11502-7. doi:10.1073/pnas.1509120112.

[33] Tang Y, Lin G, Han L, Qiu S, Yang S, Yin J. Design of Hierarchically Cut Hinges for Highly Stretchable and Reconfi gurable Metamaterials with Enhanced Strength. Adv Mater 2015:1-10. doi:10.1002/adma.201502559.

[34] Mizzi L, Attard D, Gatt R, Dudek KK, Ellul B, Grima JN. Implementation of Periodic 
Boundary Conditions for Loading of Mechanical Metamaterials and Other Complex Geometric Microstructures using Finite Element Analysis. Eng Comput 2020. doi:10.1007/s00366-019-00910-1.

[35] Materials Data Sheet - Tough Resin n.d. https://formlabsmedia.formlabs.com/datasheets/Tough_Technical.pdf (accessed December 20, 2019).

[36] Zguris Z. How the Mechanical Properties of Stereolithography 3D Prints are affected by UV Curing n.d. https://archive-media.formlabs.com/upload/How-MechanicalProperties-of-SLA-3D-Prints-Are-Affected-by-UV-Curing.pdf (accessed December 20, 2019).

[37] Mizzi L, Mahdi EM, Titov K, Gatt R, Attard D, Evans KE, et al. Mechanical metamaterials with star-shaped pores exhibiting negative and zero Poisson's ratio. Mater Des 2018;146:28-37. doi:10.1016/j.matdes.2018.02.051.

[38] Rothenburg L, Berlin AA, Bathurst RJ. Microstructure of isotropic materials with a negative Poisson's ratio. Nature 1991;354:470-2.

[39] Mizzi L, Attard D, Gatt R, Pozniak AA, Wojciechowski KW, Grima JN. Influence of translational disorder on the mechanical propertiés of hexachiral honeycomb systems. Compos Part B Eng 2015;80:84-91. doi:10.1016/j.compositesb.2015.04.057.

[40] Mizzi L, Salvati E, Spaggiari A, Tan J, Korsunsky AM. Highly stretchable twodimensional auxetic metamaterial sheets fabricated via direct-laser cutting. Int J Mech Sci 2020;167:105242. doi:10.1016/j.ijmecsci.2019.105242.

[41] Grima JN, Mizzi L, Azzopardi KM, Gatt R. Auxetic Perforated Mechanical Metamaterials with Randomly Oriented Cuts. Adv Mater 2016;28:385-9. doi:10.1002/adma.201503653.

[42] Mizzi L, Azzopardi KM, Attard D, Grima JN, Gatt R. Auxetic metamaterials exhibiting giant negative Poisson's ratios. Phys Status Solidi - Rapid Res Lett 2015;9:425-30. doi:10.1002/pssr.201510178.

[43] Wang G, Sun S, Li M, Zhou J. Large deformation shape optimization of cut-mediated soft mechanical metamaterials. Mater Res Express 2019;6:055802.

[44] Ashby MF. Materials Selection in Mechanical Design. 3rd ed. Burlington Massachusetts: Butterworth-Heinemann; 1999. 
[45] Ruzzene M, Tsopelas P, Asce AM. Control of Wave Propagation in Sandwich Plate Rows with Periodic Honeycomb Core 2003:975-86.

[46] Imbalzano G, Tran P, Ngo TD, Lee PVS. A numerical study of auxetic composite panels under blast loadings. Compos Struct 2016;135:339-52. doi:10.1016/j.compstruct.2015.09.038.

[47] Spadoni A, Ruzzene M. Static aeroelastic behavior of a chiral-core airfoil. 16th Int Conf Adapt Struct Technol 2005.

[48] Airoldi A, Bettini P, Panichelli P, Oktem MF, Sala G. Chiral topologies for composite morphing structures - Part I: Development of a chiral rib for deformable airfoils. Phys Status Solidi Basic Res 2015;252:1435-45. doi:10.1002/pssb.201451689.

[49] Scarpa F, Jacobs S, Coconnier C, Toso M, Di Maio D, Auxetic shape memory alloy cellular structures for deployable satellite antennas: design, manufacture and testing. EPJ Web Conf 2010;6:27001. doi:10.1051/epjconf/20100627001.

[50] Jacobs S, Coconnier C, Dimaio D, Scarpa F, Toso M, Martinez J. Deployable auxetic shape memory alloy cellular antenna demonstrator: Design, manufacturing and modal testing. Smart Mater Struct 2012;21. doi:10.1088/0964-1726/21/7/075013. 